\title{
Macrophages, as a Promising Strategy to Targeted Treatment for Colorectal Cancer Metastasis in Tumor Immune Microenvironment
}

\author{
Yingru Zhang ${ }^{1 \dagger}$, Yiyang Zhao ${ }^{1+}$, Qi $\mathrm{Li}^{12^{* *}}$ and Yan Wang ${ }^{1 *}$ \\ 1 Department of Medical Oncology, Shuguang Hospital, Shanghai University of Traditional Chinese Medicine, \\ Shanghai, China, ${ }^{2}$ Academy of Integrative Medicine, Shanghai University of Traditional Chinese Medicine, Shanghai, China
}

\section{OPEN ACCESS}

Edited by:

Fernando Aranda,

Instituto de Investigación Sanitaria de

Navarra (IdiSNA), Spain

Reviewed by:

Ruben Hernandez-Alcoceba,

University of Navarra, Spain

Eduardo Huarte,

PTC Therapeutics, United States

Javier Dotor,

DISIT Biotech Sl., Spain

*Correspondence:

Yan Wang

yanwang@shutcm.edu.cn

Qi Li

qili@shutcm.edu.cn

${ }^{t}$ These authors have contribute equally to this work

Specialty section:

This article was submitted to

Cancer Immunity

and Immunotherapy,

a section of the journal

Frontiers in Immunology

Received: 26 March 2021 Accepted: 24 June 2021

Published: 13 July 2021

Citation:

Zhang Y, Zhao Y, Li Q and Wang Y (2021) Macrophages, as a Promising

Strategy to Targeted Treatment for

Colorectal Cancer Metastasis in

Tumor Immune Microenvironment.

Front. Immunol. 12:685978.

doi: 10.3389/fimmu.2021.685978
The tumor immune microenvironment plays a vital role in the metastasis of colorectal cancer. As one of the most important immune cells, macrophages act as phagocytes, patrol the surroundings of tissues, and remove invading pathogens and cell debris to maintain tissue homeostasis. Significantly, macrophages have a characteristic of high plasticity and can be classified into different subtypes according to the different functions, which can undergo reciprocal phenotypic switching induced by different types of molecules and signaling pathways. Macrophages regulate the development and metastatic potential of colorectal cancer by changing the tumor immune microenvironment. In tumor tissues, the tumor-associated macrophages usually play a tumor-promoting role in the tumor immune microenvironment, and they are also associated with poor prognosis. This paper reviews the mechanisms and stimulating factors of macrophages in the process of colorectal cancer metastasis and intends to indicate that targeting macrophages may be a promising strategy in colorectal cancer treatment.

Keywords: colorectal cancer, metastasis, macrophages, targeted treatment, signaling pathways

\section{INTRODUCTION}

Colorectal cancer (CRC) is the third most common malignancy and the second leading cause of cancer deaths in the world (1). Approximately $50 \%$ of CRC patients develop metastatic diseases (2). CRC has a high probability of metastasis following its diagnosis $(3,4)$, and metastases especially involve the liver (5). Cytotoxic drug chemotherapy is usually the first choice for metastatic CRC (mCRC), but it is accompanied by numerous side effects, and the prognosis is unsatisfactory. Targeted therapy also currently plays a significant role in the treatment of mCRC, while chemotherapy combined with targeted therapy for mCRC is the first-line therapy in clinical treatment $(6,7)$. Due to the advantages of targeted therapy, such as precision, high efficiency, significantly less toxicity than chemotherapy, and convenient oral administration (8), targeted therapy will become a promising treatment approach. The discovery of novel molecular biomarkers will likely be of great significance for the treatment of $\operatorname{mCRC}(9,10)$. Immune checkpoint inhibitors, including anti-programmed cell death 1 (PD-1), anti-programmed cell death ligand 1 (PD-L1) 
monoclonal antibodies (MAbs) and CTL-associated antigen 4 (CTLA4) blockade have been confirmed to improve the overall survival rate of patients in different cancer types $(11,12)$.

The tumor immune microenvironment (TIME) is complex and diverse and mainly includes tumor cells, immune cells, antigens, and cytokines (13). Recently, investigations regarding the TIME have received great interest. Macrophages are one of the most important cells in the TIME. Macrophages promote or inhibit tumor invasion and metastasis through the interaction between various molecules and signaling pathways (14-16). Studies have shown that the high immunoactivity defined by the microsatellite instability (MSI) subtype CRC is associated with the high degree of infiltration of M1 macrophages (17). Therefore, molecular targeted therapy directed at the CRC immune microenvironment is a focal point in the treatment of tumors; targeting macrophages potentially exerts long-term effects for the treatment of mCRC.

\section{TUMOR IMMUNE MICROENVIRONMENT}

The TIME is formed by the interaction between tumor cells, tumor-infiltrating immune cells, epithelial cells, fibroblasts, blood vessels, chemokines, and cytokines $(18,19)$. In the TIME, adaptive immune effector T cells (20), and innate immunity effector cells, macrophages (21), NK cells (22), and other cells promote the inflammatory response and are involved in antitumor effects. In contrast to antitumor immunity, tumorassociated macrophages (TAMs) (23), and myeloid suppressor cells (MDSCs) (24), and regulatory $\mathrm{T}$ cells (Tregs) (25) as immunosuppressive cells also play an immunomodulatory effect in tumor immunity, facilitating the metastasis of tumors. The occurrence and development of tumors are closely associated with antitumor immune cells in the TIME. Conversely, immune cells can also be influenced by products of tumor cells such as cytokines (26) and exosomes (27), and integrate with signaling pathways by activating immune evasion mechanisms, which further induce tumor metastasis.

In the TIME associated with CRC, various immune cells interact with each other to promote or inhibit the growth, invasion, and metastatic potential of CRC (Figure 1). Dendritic cells (DCs) activate T cells through the combination of the major histocompatibility complex (MHC) and T cell receptors (28). NK cells can kill tumor cells directly through antibody-dependent cellmediated cytotoxicity (ADCC) (29), and T cells can kill tumor cells directly through cytotoxicity (30). Both NK cells and T cells can kill tumor cells through the Fas/FasL pathway, the perforingranzyme pathway (31), and by releasing tumor necrosis factor (TNF) (32). MDSCs can mediate the development of M2 macrophages and Tregs, which depend on IL-10 $(33,34)$. T cells promote tumor immunity by secreting IFN- $\gamma$ (35), while Tregs inhibit the immune effects of T cells via the PD-1/PD-L1 axis (36). Macrophages can promote or inhibit tumor immunity by polarizing into different types and play a critical role in the tumor microenvironment (TME). Furthermore, mutual transformation of macrophages regulates the TIME in CRC.

\section{THE ROLE OF MACROPHAGES IN THE METASTASIS OF CRC}

Macrophages can differentiate into different subtypes in the TIME and may play dual roles (37). They produce various molecules that interact with other immune cells and tumor cells and further affect the progression in CRC (38). Under the influence of different cytokines and exosomes, three types of macrophages can be identified: naive M0, the M1 subtype with pro-inflammatory effects, and the M2 subtype with immunosuppressive effects $(39,40)$, which promote or inhibit the progression of CRC.

\section{Antitumor Effects of Macrophages}

The classical activated macrophages are defined by the M1 macrophages whose surface markers are CD86, iNOS, and TNF- $\alpha$ (41-43), M0 macrophages polarize into M1 macrophages under the effect of LPS and IFN- $\gamma(44)$, which are active against pathogen infection and play a significant function in immunity. M1 macrophages can inhibit tumor development by releasing tumor-suppressing molecules, including TNF- $\alpha$ (45). M1 macrophages also induce tumor cell apoptosis through phagocytosis (46), ADCC (47), and release of TNF and nitric oxide (NO) (48). Studies have shown that M1-type macrophages can promote tumor immunity by recruiting cytotoxic T cells (49).

\section{Tumor-Promoting Effects of Macrophages}

Macrophages in the TIME are often called TAMs; M0 macrophages are mostly polarized into M2 macrophages (50) following induction by IL-4, IL-10, and IL-13 (51-53). CD163, CD206 and Arg1 are common surface markers of M2 macrophages (54-56). TAMs are classified as M2 macrophage activation subtype, which can promote the development of CRC in the TIME (57). A research showed that TAMs increased significantly in hepatic metastatic tumors of colorectal cancer (58). TAMs contribute to angiogenesis (59), promote epithelialmesenchymal transition (EMT) of tumor cells (60), and activate immunosuppression (61), promoting the metastasis of CRC. Comfortingly there is evidence that macrophages have plasticity $(62,63)$, and various stimuli, including drugs or M1 exosomes, can cause macrophages to alter their phenotype and reprogram M2 toward M1, inhibiting tumor development (64, 65). Therefore, targeting macrophages will be a potential strategy for the treatment of colorectal cancer metastasis.

\section{SIGNALING PATHWAYS INVOLVING MACROPHAGES IN CRC METASTASIS}

The immune cells in the TIME regulating CRC development are achieved by the secreted immune molecules and CRC cellsurface receptors, activating the intracellular signaling pathways involving macrophages, including $\mathrm{Wnt} / \beta$-catenin, NF- $\kappa$ B, PI3K/AKT, JAK/STAT3, MAPK, and TGF- $\beta /$ Smad signaling pathways (Figure 2). 


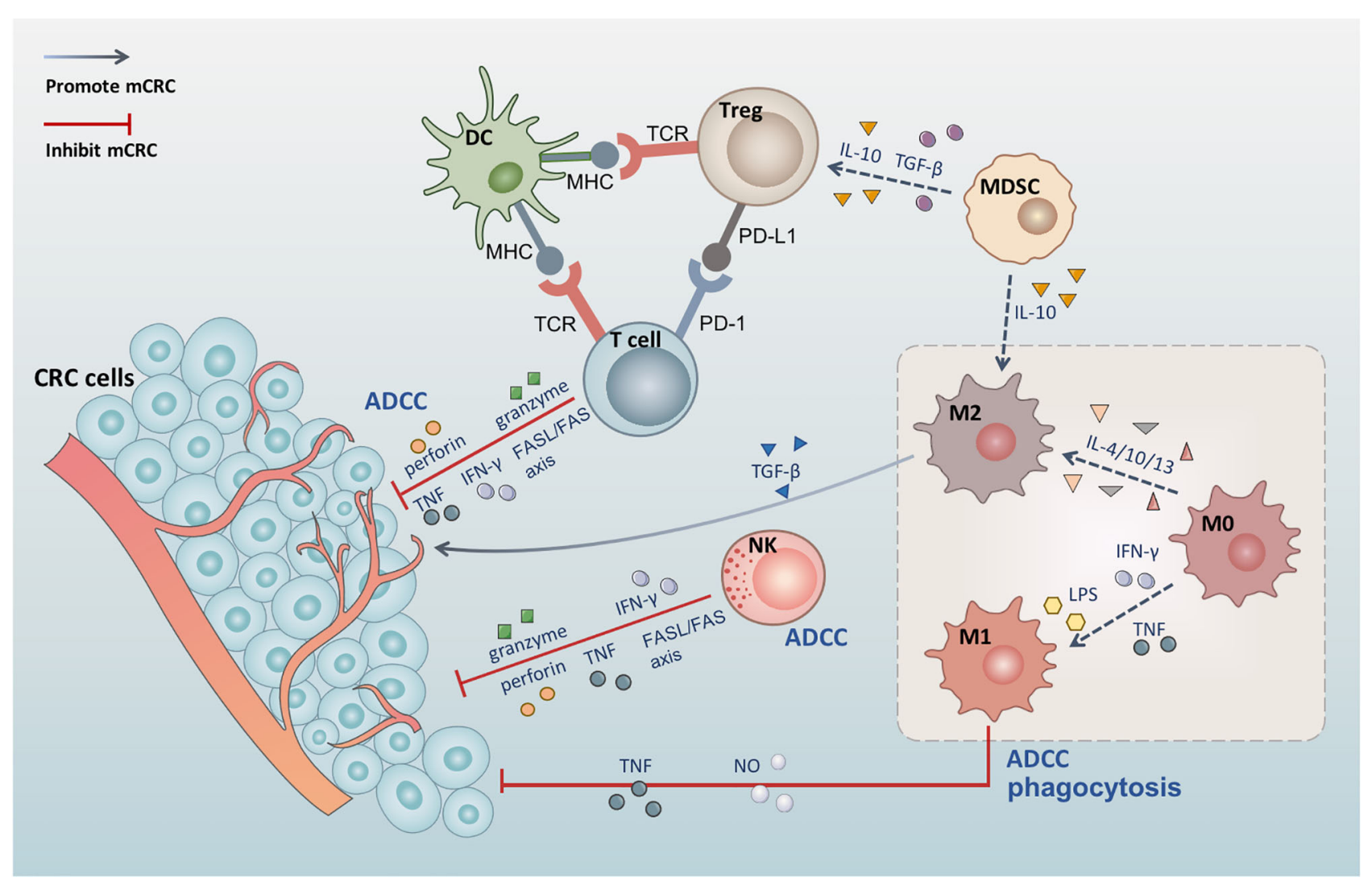

FIGURE 1 | The tumor immune microenvironment of colorectal cancer. DC activates T cells through the combination of the MHC and T cell receptors. Tregs inhibit the immune effects of T cells via the PD-1/PD-L1 axis. Both NK cells and T cells can kill tumor cells through the Fas/FasL pathway, the perforin-granzyme pathway, and by releasing TNF and IFN- $\gamma$. MDSCs can mediate the development of M2 macrophages and Tregs, which depends on IL-10, and produce TGF- $\beta$ to induct Tregs. MO macrophages polarize into M1 macrophages under the effect of LPS, IFN- $\gamma$, and TNF, while polarizing into M2 macrophages following induction by IL-4, IL-10, and IL-13. M1 macrophages also induce tumor cell apoptosis through phagocytosis, ADCC, and the release of TNF and NO. M2 secretes TGF- $\beta$ and contributes to angiogenesis, promotes tumor cell EMT, and induces immunosuppressive microenvironment.

\section{Wnt/ $\beta$-Catenin Signaling Pathway}

The wnt/ $\beta$-catenin signaling pathway promotes the development of CRC by regulating the growth, differentiation, and migration of tumor cells (66). Abnormalities of the wnt signaling, such as the APC gene mutation, are common in CRC, which can promote the development of CRC (67). A global transcriptome immune classification experiment for CRC solid tumors showed that most patients had APC mutations (68). Activation of the wnt signaling pathway leads to granulocyte recruitment and tumor invasion, and abnormal wnt signaling directly alters the antineoplastic activities of effector $\mathrm{T}$ cells, helper $\mathrm{T}$ cells, and Tregs, suppressing tumor immunity (69). In highly proliferative colorectal tumors, wnt $/ \beta$-catenin signaling is activated and abundant $\beta$-catenin accumulates in the nucleus, accompanying the immune cell infiltrates including TAMs (70).

\section{NF- $\kappa B$ Signaling Pathway}

Nuclear factor- $\kappa \mathrm{B}(\mathrm{NF}-\kappa \mathrm{B})$ is a critical molecule underlying the relationship between inflammation and tumor immunity and is involved in the growth and development of CRC (71). Various extracellular factors including proinflammatory cytokines, LPS, and growth factors lead to $\mathrm{I} \kappa \mathrm{B}$ protein phosphorylation and ubiquitination and then degradation, freeing NF- $\kappa \mathrm{B} / \mathrm{Rel}$ complexes and transferring into the nucleus for transcription, promoting EMT, angiogenesis, and metastasis $(72,73)$.

Transcription factors mediated by NF- $\kappa B$ are associated with MDSC activation (74). NF- $\kappa \mathrm{B}$ expression is upregulated in CD4+TIM-3+ tumor-infiltrating lymphocytes, inducing the expression of inflammatory factors and $\mathrm{T}$ cell failure, which in turn further facilitates the metastasis of CRC (75). The NF- $\kappa \mathrm{B}$ pathway is activated in P2X7R overexpressed CRC cells, cytokines increasing leads to the recruitment of TAMs (76). TAMs can significantly upregulate the vascular endothelial growth factor-A (VEGF-A) of CRC cells and activate the NF$\kappa \mathrm{B}$ signaling pathway by secreting IL-6 and IL-8, promoting CRC metastasis (77). In patients with CRC, high levels of p50NF- $\kappa B+$ TAMs at the invasive margin are associated with poor prognosis following surgical intervention for excision of tumors. The p50-NF- $\mathrm{KB}+$ TAMs participate in the development of CRC by reducing recruitment and antitumor activity of $\mathrm{T}$ cells, which confirms that the NF- $\kappa \mathrm{B}$ pathway is a significant signaling pathway promoting CRC metastasis (78). However, the role of 


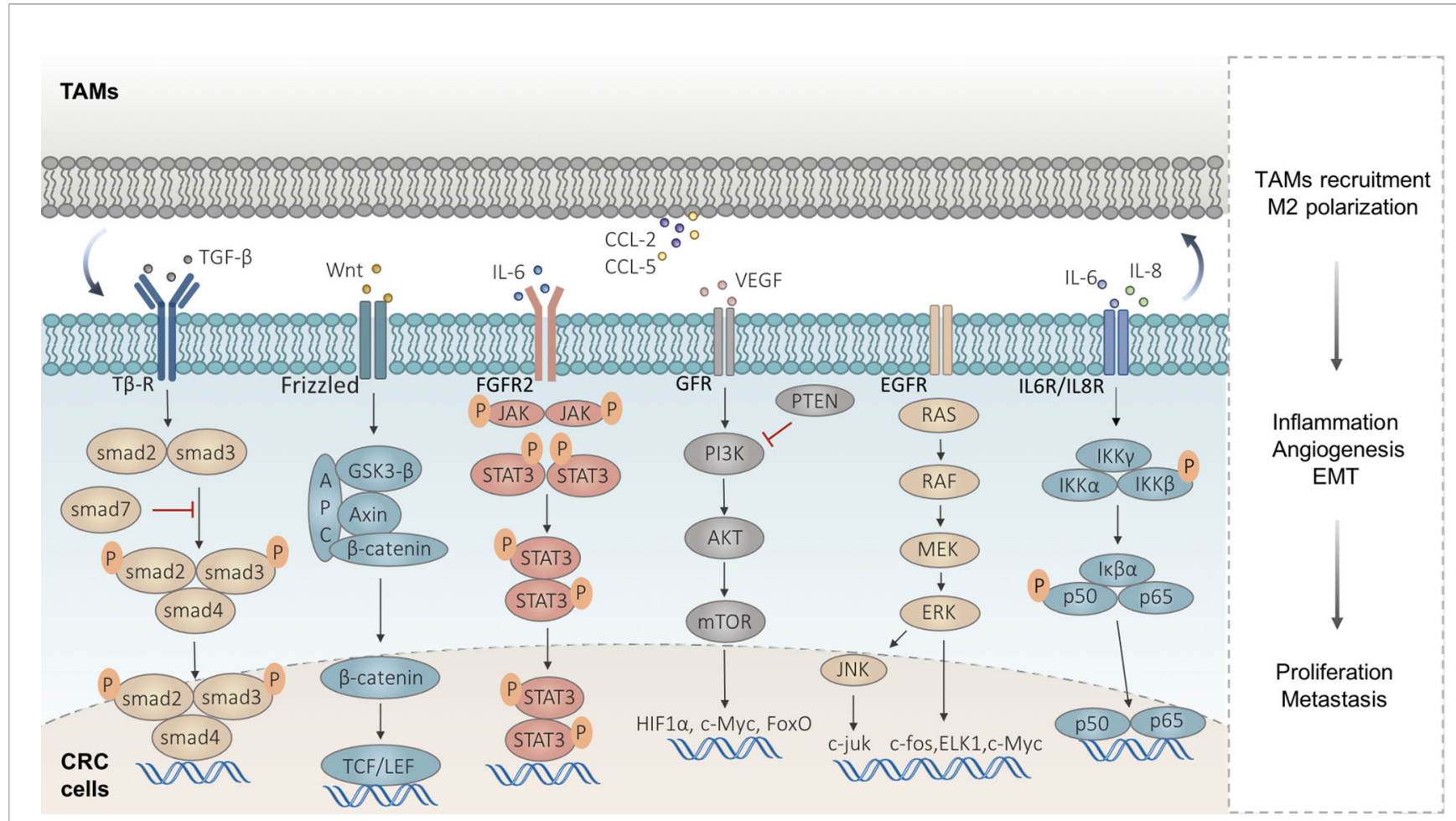

FIGURE 2 | Signaling pathways implicated in macrophages in CRC metastasis. Wnt/ $\beta$-catenin signaling is activated in highly proliferative CRC accompanied by the TAM infiltrates. TAMs upregulate the VEGF-A of CRC cells and activate the NF- $\kappa$ B signaling pathway by secreting IL-6 and IL-8. TAMs produce IL-6 to activate the JAK/STAT3 signaling in CRC cells and lead to the EMT, which in turn lead to the CCL2/5 secreted by CRC cells, to promote macrophage recruitment. Some exosomes carrying miRNAs derived from CRC cells regulate PTEN, by activating the PI3K/Akt signaling to induce M2-like TAM polarization, and in turn, TAMs promote CRC metastasis by enhancing EMT and secreting VEGF. The abnormal activation of the MAPK signaling pathway can induce tumor cell proliferation and participates in the development and metastasis of CRC. TAMs facilitate the EMT program involved in the CRC metastatic process via TGF- $\beta /$ Smad signaling.

$\mathrm{NF}-\kappa \mathrm{B}$ in colorectal cancer remains controversial. A research found that for macrophages, the activation of NF- $\kappa \mathrm{B}$ can promote M1-polarization, so as to play an antitumor effect (79).

\section{JAK/STAT3 Signaling Pathway}

In the TIME, the abnormal activation of the JAK/STAT3 signaling contributes to an immunosuppressive tumor microenvironment, which promotes tumor growth and metastasis (80). Studies have indicated that TAMs produce pro-inflammatory cytokine IL-6 to activate the JAK/STAT3 signaling in CRC cells and lead to epithelial-mesenchymal transition (EMT) involved in tumor progression (81), which in turn leads to the CCL2 secreted by CRC cells, to promote macrophage recruitment, while, inhibition of CCL2 or IL6 can break this crosstalk (82).

On the other hand, IL-6 can upregulate other inflammatory factors such as CCL2 and CCL5, and then recruit macrophages $(83,84)$. Furtherly, the recruited macrophages in turn secret IL-6 to activate JAK2/STAT3 signaling, promoting tumor metastasis (85). CCL2 significantly increases the number of MDSCs and M2-like TAMs mediated by STAT3, suppresses T cells, and promotes immune evasion in CRC (86). All the above studies have shown that CRC cells and TAMs influence each other to promote tumor development.

\section{Phosphatidylinositol 3 Kinase/AKT Signaling Pathway}

The phosphatidylinositol 3 kinase (PI3K)/AKT signaling pathway is one of the most activated pathways in human cancer $(87,88)$, which can regulate the immunosuppressive microenvironment, promoting immune cell exhaustion and inhibiting antitumor activity (89). PD-1/PD-L1 blockade can rescue depleted CD8+ T cells via the PI3K/Akt/mTOR signaling pathway (90). Tumor cells overexpressing T cell immunoglobulin mucin-4 (TIM-4) activate $\mathrm{PI} 3 \mathrm{~K} / \mathrm{AKT} / \mathrm{mTOR}$ signal transduction and recruit TAMs, promoting proliferation and tumor matrix remodeling in CRC (91). Some exosomes carrying miRNAs (miR-25-3p, miR-130b$3 p$, miR-425-5p) derived from CRC cells regulate PTEN by activating $\mathrm{PI} 3 \mathrm{~K} / \mathrm{Akt}$ signaling to induce M2-like TAM polarization, and in turn, TAMs promote CRC metastasis by enhancing EMT and secreting vascular endothelial growth factor (VEGF) (92). In addition, inhibition of AKT can effectively limit the differentiation of $\mathrm{T}$ cells and enhance antitumor effects in vivo (93).

\section{MAPK Signaling Pathway}

The mitogen-activated protein kinase (MAPK) signaling pathway is one of the most important bridges for converting extracellular signals to intracellular responses (94). As the key 
pathway participating in cell proliferation, the abnormal activation of the MAPK signaling pathway can induce tumor cell proliferation and participate in the development and metastasis of CRC $(95,96)$. The homeoprotein Six1 is associated with poor prognosis in CRC (97). Six 1 overexpression promotes CRC growth and metastasis by stimulating angiogenesis and recruiting TAMs, accompanied by MAPK activation in CRC cells (98). In addition, a study demonstrated that TAMs interact with CRC cells inducing EMT in CRC cells by activating the MAPK pathway in TAMs, and then promoting the metastasis of CRC (77). The crosstalk in TAMs and CRC cells reveals the significant role of TAMs in the development of CRC, which provides a powerful argument for targeting TAMs in CRC treatment.

\section{TGF- $\beta /$ Smad Signaling Pathway}

In tumor stroma, M2-polarized TAMs secrete transforming growth factor-beta (TGF- $\beta$ ) via the miR-34a/VEGF axis and promote invasion and metastasis of CRC (99). There are also some studies that found that in vitro, cytokines induced M2 macrophages to produce TGF- $\beta 1$ via the VEGF/VEGFR2 signaling pathway (57). TAMs facilitate the EMT program involved in the CRC metastatic process via TGF- $\beta / \operatorname{Smad} 2,3-4 /$ Snail signaling $(100,101)$. A multicolor histology analysis indicated that patients with poor clinical outcomes may also have infiltration of $\mathrm{T}$ cells in tumor tissues, but always with high TGF- $\beta$ expression and high TAM density (102), which reveals the critical role of TAMs in CRC metastasis.

Generally, in the immune microenvironment, TGF- $\beta$ and IL6 are required for the development of Th-17 cells which produce IL-17 (103). The production of IL-17 is positively correlated with distant colon tumorigenesis (104). However, different from the local tumor immune response, studies have found that in nontumor tissues of cancer patients, the increase of Th-17 cells and IL-17 can enhance M1 polarization while inhibiting M2 polarization (105), which indicates the complex role of TGF $\beta$ in human immunity.

\section{MOLECULES RELATED TO MACROPHAGES IN CRC METASTASIS}

Molecular targeted therapy is a promising method for CRC therapy, especially in mCRC. Macrophages play a pivotal role in the metastatic niche, and many molecules, including IL-4, IL10 , and IL-13, can promote macrophages to polarize into M2. In turn, M2 can secrete IL-6, IL-8, and other inflammatory factors to promote the proliferation and metastasis of cancer cells. Therefore, macrophages act as important mediators of the development of CRC. Targeting macrophages may provide a new strategy for the treatment of CRC. All molecules related to targeting macrophages are listed in Table $\mathbf{1 .}$

\section{Non-Coding RNA}

Studies have demonstrated that non-coding RNA molecules play a pivotal role in the polarization process of TAMs. The overexpression of the long non-coding RNA (LncRNA) RPPH1 has been associated with advanced tumor-node-metastasis (TNM) stages and poor prognosis (106). Exosomes derived from CRC cells transport RPPH1 into macrophages and mediate M2-like polarization to promote CRC cell proliferation and metastasis (107). The lncRNA HLA-F-AS1 regulates the expression of profilin 1 (PFN1) in CRC-derived EVs by inhibiting miR-375, and then, in turn, mediates the M2 phenotype polarization of macrophages (108), promoting the CRC metastasis.

Several miRNAs including miR-25-3p, miR-425-5p, and miR-130b-3p induce macrophage M2 polarization by activating the CXCL12/CXCR4 axis in CRC metastasis (92). The tumor-derived exocrine miR-934 can promote CRC liver metastasis by regulating the interaction between CRC cells and TAMs (110). In addition, exosomes carrying miRNA-106b-5p promote the M2-like polarization of macrophages and induce the EMT in CRC cells, which is implicated in the crosstalk between tumor cells and TAMs (131). Brahma-related gene-1 (BRG1) is the core subunit of switch/sucrose nonfermentable (SWI/SNF) family complexes (132). M2-macrophages-derived exosomes carry miR-155-5p and miR-21-5p to CRC cells and combine with the BRG1 coding sequence to downregulate the expression of BRG1, promoting the metastasis of CRC (109).

Thus, we can achieve the effect of blocking M2-like polarization or of blocking tumor from promoting secretion from TAMs by inhibiting the above non-coding RNA, thereby controlling the tumor-promoting effect of macrophages in the tumor microenvironment of CRC.

\section{Cytokines}

Many cytokines are involved in the polarization of TAMs, some of them active in downstream signaling pathways to promote CRC metastasis. Studies have revealed that complex chemokine networks can affect cancer progression via the recruitment and activation of TAMs. The increased expression of CCL17 in DCs and M2-like TAMs in tumors induces an immunosuppressive environment; CCL17 expression has been used as a marker for M2-like immunosuppressive macrophage polarization (133). CCL5, secreted by TAMs, inhibits T-cell-mediated killing of CRC cells and promotes immune escape by stabilizing PD-L1 (113). In addition, CRC cells secrete VEGF-A and then stimulate TAMs to produce CXCL1 in primary tumors. The increased release of CXCL1 transfer to the liver via blood circulation recruits CXCR2-expressing MDSCs to form a pre-metastatic niche, promoting liver metastasis (114).

In addition, sST2, a soluble isoform of the IL-33 receptor (ST2), suppresses angiogenesis, macrophage infiltration, and macrophage M2 polarization induced by IL-33 (111). M2polarized TAMs secrete TGF- $\beta$ (100), which regulates the miR34a/VEGF axis to facilitate CRC cell proliferation and invasion (99). Wnt5a is highly expressed in TAMs and can induce M2 polarization by regulating the secretion of IL-10, which is mediated by the CaMKII-ERK1/2-STAT3 pathway (117). Furthermore, Wnt5a ${ }^{+}$TAMs promote CRC development which also depends on CCL2 secretion mediated by the CaMKII-ERK pathway (112). 
TABLE 1 | Molecules related to TAMs in CRC metastasis.

\begin{tabular}{|c|c|c|c|c|c|}
\hline Molecules & Types & $\begin{array}{l}\text { Expression in } \\
\text { mCRC }\end{array}$ & Mechanism & $\begin{array}{l}\text { Effects in } \\
\text { CRC }\end{array}$ & References \\
\hline IncRNA & Non-coding RNA & Up & Mediates the polarization of M2 & Promote & $(106,107)$ \\
\hline \multicolumn{6}{|l|}{ RPPH1 } \\
\hline IncRNA HLA- & Non-coding RNA & Up & Mediates the polarization of M2 & Promote & $(108)$ \\
\hline \multicolumn{6}{|c|}{ F-AS1 } \\
\hline miR-21-5p & Non-coding RNA & Up & Derived by M2-macrophages, downregulates the expression of BRG1 & Promote & $(109)$ \\
\hline miR-25-3p & Non-coding RNA & Up & Activates CXCL12/CXCR4 axis, induces M2 polarization & Promote & $(92)$ \\
\hline miR-130b-3p & Non-coding RNA & Up & Activates CXCL12/CXCR4 axis, induces M2 polarization & Promote & (92) \\
\hline miR-155-5p & Non-coding RNA & Up & Derived by M2-macrophages, downregulates the expression of BRG1 & Promote & $(109)$ \\
\hline miR-425-5p & Non-coding RNA & Up & Activates CXCL12/CXCR4 axis, induces M2 polarization & Promote & $(92)$ \\
\hline miR-934 & Non-coding RNA & Up & $\begin{array}{l}\text { Promotes CRC liver metastasis by regulating the interaction between CRC } \\
\text { cells and TAMs }\end{array}$ & Promote & $(110)$ \\
\hline sST2 & Cytokine receptor & Down & $\begin{array}{l}\text { Suppresses IL-33-induced angiogenesis, macrophage infiltration and } \\
\text { polarization }\end{array}$ & Inhibit & $(111)$ \\
\hline CCL2 & Cytokines & Up & Promotes the recruitment of macrophages & Promote & $\begin{array}{l}(82,86,98 \\
112)\end{array}$ \\
\hline CCL5 & Cytokines & Up & Promotes the recruitment of macrophages & Promote & $(83,113)$ \\
\hline CCL17 & Cytokines & Up & Upregulates in M2-like TAMs, induces an immunosuppressive environment & Promote & $(111)$ \\
\hline CXCL1 & Cytokines & Up & Secreted by TAMs, forms a pre-metastatic niche, promotes liver metastasis & Promote & $(114)$ \\
\hline TGF- $\beta$ & Cytokines & Up & Secreted by TAMs, facilitates EMT in CRC & Promote & $(101)$ \\
\hline VEGF & Cytokines & Up & Augments the recruitment of TAMs & Promote & $\begin{array}{c}(98,99,115 \\
116)\end{array}$ \\
\hline CSF-1 & Cytokines & Up & Augments the recruitment of TAMs & Promote & (98) \\
\hline IL-1 $1 \beta$ & Cytokines & Up & Regulates the crosstalk between TAMs and CRC cells & Promote & $(115,116)$ \\
\hline IL-6 & Cytokines & Up & Upregulates CCL2 and CCL5, and then recruits TAMs & Promote & $(81)$ \\
\hline IL-10 & Cytokines & Up & Induces the M2 polarization & Promote & $(117)$ \\
\hline LUM & Metabolites & Up & Regulates macrophage polarization & Promote & $(118)$ \\
\hline ABHD5 & Metabolites & Down & Low-level expressed in migratory TAMs, upregulates the MMPs & Promote & $(119)$ \\
\hline PRL-3 & Phosphatases & Up & Activates the MAPK pathway in TAMs to promote EMT & Promote & $\begin{array}{l}(77,120 \\
121)\end{array}$ \\
\hline Shp2 & Phosphatases & Up & Promotes the maturation of TAMs & Promote & $(122)$ \\
\hline KRS & proteases & Up & Induces M2 polarization of macrophages & Promote & $(122)$ \\
\hline CTSK & proteases & Up & Induces M2 polarization of macrophages & Promote & $(123)$ \\
\hline Gas6 & protein & Up & Induces M2 polarization of macrophages & Promote & $(122)$ \\
\hline NLRC4 & Inflammasome & Up & Regulates the crosstalk between TAMs and CRC cells & Promote & $(116)$ \\
\hline NLRP3 & Inflammasome & Up & Regulates the crosstalk between TAMs and CRC cells & Promote & $(115)$ \\
\hline Wnt5a & Secreted protein & Up & Activates macrophages polarization & Promote & $(112,117)$ \\
\hline S100A8 & $\begin{array}{l}\text { Calcium- and zinc- } \\
\text { binding protein }\end{array}$ & Up & Activates the $\mathrm{NF}-\kappa \mathrm{B}$ pathway in macrophages & Promote & $(124)$ \\
\hline GRP78 & $\begin{array}{l}\text { Glucose regulated } \\
\text { protein }\end{array}$ & Up & Upregulates by TAMs, promotes STAT3 phosphorylation & Promote & $(125)$ \\
\hline P2X7R & Purine receptor & Up & Leads to the recruitment of TAMs via NF- $\kappa$ B pathway & Promote & $(76,126)$ \\
\hline LAYN & Hyaluronan receptor & Up & $\begin{array}{l}\text { Activates macrophages polarization and associates with poor prognosis of } \\
\text { patients }\end{array}$ & Promote & $(127)$ \\
\hline cox-2 & Cyclooxygenase & Up & $\begin{array}{l}\text { Promotes the differentiation of M2 macrophages and reduces the } \\
\text { expansion of } \mathrm{M} 1 \text { macrophages }\end{array}$ & Promote & (128) \\
\hline PGE2 & Prostaglandin E2 & Up & $\begin{array}{l}\text { Promotes the differentiation of M2 macrophages and reduces the } \\
\text { expansion of } \mathrm{M} 1 \text { macrophages }\end{array}$ & Promote & $(128,129)$ \\
\hline IDO & $\begin{array}{l}\text { Indoleamine } 2,3- \\
\text { dioxygenase } 1\end{array}$ & Up & $\begin{array}{l}\text { Promotes Tregs and M2 macrophages cooperative effects, leads to } \\
\text { immunosuppression }\end{array}$ & Promote & (130) \\
\hline KYN & Kynurenine & Up & $\begin{array}{l}\text { Promotes Tregs and M2 macrophages cooperative effects, leads to } \\
\text { immunosuppression via AHR axis }\end{array}$ & Promote & $(130)$ \\
\hline
\end{tabular}

Therefore, we can regulate the immunosuppressive microenvironment by inhibiting the cytokine-induced macrophage M2-like polarization, decrease the recruitment of immunosuppressive cells, and enhance tumor immunity for CRC metastasis.

\section{Macrophage-Related Metabolites}

Many metabolites are also related to the metastasis of CRC. These factors are involved in tumor progression and offer a new direction for mCRC treatment. A small leucine-rich proteoglycan lumican (LUM) regulates macrophage polarization in colorectal adenocarcinoma and induces immune escape in the microenvironment of CRC (118). TAMs play an important role in tumor invasion and can migrate with tumor cells during the process of tumor metastasis. TAMs exhibit a heterogeneous expression of the hydrolase domain containing the triglyceride hydrolytic activator 5 (ABHD5) which is expressed in low-level in migratory TAMs and 
upregulate matrix metalloproteinases (MMPs) involved in CRC metastasis (119). According to the above, we consider that the regulation of Lum or ABHD5 can target TAMs to prevent the metastasis of CRC.

\section{Phosphatases and Proteases}

During the metastasis of CRC, phosphatases and proteases play an important role. It has been found that the protein tyrosine phosphatase-3 (PRL-3) (120) increases CCL26 secretion to stimulate TAM infiltration (121) and activates the MAPK pathway in TAMs, ultimately initiating EMT. On the other hand, PRL-3 can directly induce angiogenesis via NF- $\kappa \mathrm{B}$ signaling (77). Tyrosine phosphatase 2 (Shp2), which contains two homologous domains of $\mathrm{Src}$, is a non-receptor tyrosine phosphatase encoded by the PTPN11 gene and is positively correlated with tumor metastasis. Studies have shown that Shp2 promotes the maturation of TAMs by activating RAS, and it is associated with PD-1 signaling in T cells (134). KRASpositive CRC cells secrete cytokines, including growth arrestspecific 6 (Gas6) and cause M2 macrophages polarization and infiltration. In addition, CAFs are activated by communication between CRC cells and TAMs, which remodels the environment of CRC metastasis for cancer cell dissemination (122, 135). Cathepsin K (CTSK) is a lysosomal cysteine protease, which is implicated in signal transduction in cancer cells. CTSK, secreted by CRC cells, induces the polarization of M2 macrophages and mediates the interaction between the gut microbiota imbalance and CRC metastasis, and CTSK overexpression in CRC predicts advanced progression and poor prognosis (123). Thus, it can be seen that the downregulation of these enzymes can block the interaction between TAMs and CRC cells and then inhibit the development of CRC.

\section{Other Biomolecules}

Many other biomolecules are also involved in TAM-mediated CRC metastasis. Both nucleotide-binding oligomerization domain (NOD)-like receptor C4 (NLRC4) and NOD-like receptor family pyrin domain containing 3 (NLRP3) are the main components of the inflammasome, which can increase TAM infiltration and IL- $1 \beta$ production, and promote CRC metastasis by regulating the crosstalk between TAMs and CRC cells $(115,116)$.

The P2X purine receptor 7 (P2X7R) expressed in tumors leads to the recruitment of TAMs via the NF- $\kappa \mathrm{B}$ pathway, which facilitates the angiogenesis and the progression of CRC $(76,126)$. Tumor cells characterized by the overexpression of homologous protein Sixl can raise the recruitment of TAMs by increasing the expression of CSF-1, CCL2, CCL5, and VEGF, promoting CRC metastasis (98). S100 calcium-binding protein A8 (S100A8) can activate NF- $\kappa \mathrm{B}$ signaling in macrophages and upregulate IL- $1 \beta$ and TNF- $\alpha$ in TME and augment the migration of CRC cells (124). LAYN, a cell surface hyaluronan (HA) receptor, may be used as a prognostic biomarker for CRC, and it is associated with immune infiltration including TAMs (127). Complement 5a expressed in CRC cells activates macrophage polarization, which in turn facilitates CRC liver metastasis via the NF- $\kappa \mathrm{B}$ pathway (136).
M2-like macrophages have been reported to upregulate the expression of the glucose-regulated protein of $78 \mathrm{kDa}$ (GRP78) in tumor cells, promoting STAT3 phosphorylation, leading to the downstream inflammatory factors including IL-1 $\beta$ and TNF$\alpha$ upregulation, which facilitates tumor progression (137).

COX-1 and COX-2 are two isozymes of cyclooxygenase (COX). COX-2 has been found in high levels in CRC (125). Studies have confirmed that COX-2 is a promoting factor for liver metastasis of CRC, and it can convert arachidonic acid into prostaglandin E2 (PGE2) $(138,139)$. TAMs are the main source of COX-2 in intestinal tumors; PGE2-bound EP4 promotes the differentiation of immunosuppressive M2 macrophages and reduces the expansion of immunostimulatory M1 macrophages (128). PGE2 also enhances the tumor infiltration of M2 macrophages and promotes the development and metastasis of CRC (129).

In addition, Indoleamine 2,3-dioxygenase 1 (IDO) suppresses $\mathrm{T}$ cell immunity by catabolizing tryptophan into kynurenine (KYN) and promotes CD8+ T cell exhaustion (140). In IDOexpressing tumors, Tregs cooperate with M2-like macrophages, promoting immune suppression via Kyn-aryl hydrocarbon receptor (AHR) axis (130). Studies have shown that in tumor tissues, the levels of IDO1 and its catabolite KYN are higher in late stages (stages III and IV) than in early stages (stages I and II) of CRC patients (141). Also, IDO was found to be negatively correlated with the survival rate of patients (142).

From the above data, we infer that by interfering with these molecules in TIME, we can directly or indirectly block the crosstalk between TAMs and CRC cells and then inhibit the progression of the tumor.

\section{CLINICAL DEVELOPMENT OF TARGETED THERAPY IN CRC METASTASIS}

In recent years, the development and applications of clinical targeted drugs have been increasing. In the treatment of CRC metastasis, targeted drugs that have entered clinical application stage or clinical trials include bevacizumab, ramucirumab, cetuximab, panitumumab, trastuzumab, regorafenib, lapatinib, erlotinib, napabucasin, sym004, and pimasertib (Table 2).

TAMs are one of the causes of tumor angiogenesis and tumor immune escape mechanisms, and targeted treatment of macrophages represents a new challenge and may become a novel strategy for cancer therapy. In the TME, the antiangiogenic drugs bevacizumab and ramucirumab can bind to human VEGF and block its biological activity (143-145); cetuximab and panitumumab bind to the epidermal growth factor receptor (EGFR), repolarize TAMs from M2-like to M1-like phenotypes, recruit myeloid effector cells such as M1 macrophages and PMN for tumor cell killing by ADCC (147149), and inhibit angiogenesis and vascular endothelial permeability (162-164), and thus block M2 cell infiltration in the inflammatory environment and impede tumor development (165-167). HER2 is positively expressed in CRC, and some studies have shown that trastuzumab and lapatinib, drugs 
TABLE 2 | Targeted drugs of the treatment in mCRC.

\begin{tabular}{|c|c|c|c|c|c|}
\hline $\begin{array}{l}\text { Targeted } \\
\text { drugs }\end{array}$ & Types & Target & Mechanism & Association with macrophage & References \\
\hline Bevacizumab & Human monoclonal lgG1 antibody & VEGF & Inhibits angiogenesis of tumor & Inhibits the infiltration of TAMs & $(143-146)$ \\
\hline Ramucirumab & Human monoclonal lgG1 antibody & VEGF & Inhibits angiogenesis of tumor & Inhibits the infiltration of TAMs & $(144,145)$ \\
\hline Cetuximab & Human monoclonal lgG1 antibody & EGFR & $\begin{array}{l}\text { Inhibits angiogenesis and vascular } \\
\text { endothelial permeability }\end{array}$ & $\begin{array}{l}\text { Repolarizes TAMs from M2-like to M1-like } \\
\text { phenotypes }\end{array}$ & $(147-149)$ \\
\hline Panitumumab & Human monoclonal lgG2 antibody & EGFR & $\begin{array}{l}\text { Inhibits angiogenesis and vascular } \\
\text { endothelial permeability }\end{array}$ & $\begin{array}{l}\text { Recruits myeloid effector cells such as M1 } \\
\text { macrophages and PMN for tumor cell killing by } \\
\text { ADCC }\end{array}$ & $(47,148)$ \\
\hline Trastuzumab & Human monoclonal lgG antibody & HER2 & Blocks the growth of cancer cells & Increases macrophage levels and phagocytosis & $(146,150)$ \\
\hline Lapatinib & Human monoclonal lgG antibody & HER2 & Blocks the growth of cancer cells & Reduces the content of TAMs in TIME & $(150,151)$ \\
\hline Regorafenib & Multi-kinase inhibitor & VEGF & Inhibits angiogenesis of tumor & $\begin{array}{l}\text { Reduces the content of TAMs, increase M1 } \\
\text { polarization of macrophages }\end{array}$ & $(152-154)$ \\
\hline Erlotinib & EGFR tyrosine kinase inhibitor & EGFR & $\begin{array}{l}\text { Blocks tumor growth by inhibiting } \\
\text { the activity of tyrosine kinase }\end{array}$ & $\begin{array}{l}\text { Reduces the content of TAMs, increases M1 } \\
\text { polarization of macrophages }\end{array}$ & $(155-157)$ \\
\hline Napabucasin & Inhibitor of STAT3 & STAT3 & $\begin{array}{l}\text { Inhibits tumor metastasis and } \\
\text { recurrence }\end{array}$ & Reduces the polarization and infiltration of $\mathrm{M} 2$ & $(158,159)$ \\
\hline Sym004 & Anti-EGFR Antibody Mixture & EGFR & $\begin{array}{l}\text { Inhibits tumor growth and } \\
\text { metastasis }\end{array}$ & Reduces the polarization and infiltration of M2 & $(160)$ \\
\hline Pimasertib & MEK inhibitor & MAPK & $\begin{array}{l}\text { Inhibits the development and } \\
\text { metastasis of CRC }\end{array}$ & Reduces the polarization and infiltration of $\mathrm{M} 2$ & $(161)$ \\
\hline
\end{tabular}

targeting HER2, can inhibit tumor formation by increasing macrophage levels and phagocytosis, and by increasing the infiltration of immune cells, it exerts a therapeutic effect on CRC metastasis $(83,146,150,151)$.

Besides the mentioned monoclonal antibodies above, there are several targeted drugs proposed for the treatment of mCRC. Regorafenib, a multi-kinase inhibitor, not only plays an antiangiogenesis role by inhibiting VEGF but also induces M2 to M1 TAM polarization (152-154). Erlotinib can inhibit the phosphorylation of intracellular tyrosine kinases associated with EGFR, reducing the content of TAMs (155-157). Napabucasin inhibits the STAT3, which is associated with tumor stemness. Due to the increasing evidence supporting the overexpression of STAT3 in CRC cells, it can be inferred that napabucasin may reduce the STAT3-mediated TAM infiltration and chemoresistance $(158,159)$. Furthermore, as previously mentioned, in the TIME, the activation of the MAPK pathway in CRC cells can promote the recruitment of TAMs. Pimasertib, a drug targeting MAPK, has also been shown to be effective in phase I clinical treatment of mCRC (161). Sym004, a dual-antibody mixture targeting non-overlapping EGFR epitopes, can inhibit the infiltration of macrophages in TIME, thus providing a good therapeutic approach for mCRC $(160,166)$. All the abovementioned pre-clinical and clinical-stage drugs are implicated in macrophage, which suggests that the development of macrophagetargeted drugs have long-term clinical significance.

\section{CLINICAL TRIAL DRUGS TARGETING TAMS IN METASTATIC CRC}

There are drugs targeting macrophages to treat mCRC in clinical trials, either as a single therapy or in combination with chemotherapy or immunotherapy (Table 3). Studies have shown that the expression of PD-1 by TAMS can inhibit the phagocytosis of macrophages against tumors and tumor immunity (173). Also, macrophage colony-stimulating factor 1 (CSF-1) plays an important role in macrophage differentiation and angiogenesis (174). In the clinical research on the treatment of $\mathrm{mCRC}$, there are many studies on the anti-CSF-1 receptor (CSF-1R) and anti-PD-1/PD-L1 targeted drugs. RG7155 (emactuzumab) is a humanized $\mathrm{mAb}$ that binds to CSF1R and blocks its dimerization. In mouse models of CRC, RG7155 treatment reduces the infiltrated TAMs and increase CD8 $(+) / C D 4(+) \mathrm{T}$ cell ratio (168). Pexidartinib and Durvalumab are anti-CSF1R and anti-PD-L1 drugs respectively. Recently, clinical studies are evaluating the safety and activity of their combination in patients with advanced/metastatic CRC and clinically active pancreatic cancer (169).

In addition, granulocyte-macrophage colony stimulating factor (GM-CSF) can enhance the function of macrophages and other immune cells and improve the antitumor and antiinfective immunity of the body (175). GM-CSF is widely used in clinical research. A clinical trial demonstrated the safety and feasibility of the GM-CSF colon cancer vaccine administered to patients with mCRC and recommended that it is necessary to further study the efficacy and antitumor immunity of this vaccine $(176,177)$. JX-594 is recombinant vaccinia granulosa cellmacrophage colony stimulating factor (RAC VAC GM-CSF). It has been proved that intravenous infusion of Pexa-Vec (JX-594) is a safe and well-tolerated drug (170). At present, a phase 2 study of Pexa-Vec combined with irinotecan in patients with mCRC is

TABLE 3 | Current clinical trial drugs targeting TAMs in mCRC treatment.

\begin{tabular}{|c|c|c|c|}
\hline Drug & Target & Inhibitor type & References \\
\hline RG7155 & CSF-1R & $\mathrm{mAb}$ & (168) \\
\hline Pexidartinib & CSF-1R & Small molecule & (169) \\
\hline JX-594 & GM-CSFR & Small molecule & $(170)$ \\
\hline GVAX & GM-CSFR & Allogeneic colon cancer cell vaccine & $(171,172)$ \\
\hline Durvalumab & PD-L1 & $\mathrm{mAb}$ & (169) \\
\hline
\end{tabular}


currently under way (178). Drugs targeting epigenomes include DNA methyltransferase 1 inhibitor (DNMTi) and histone deacetylase inhibitor (HDACi). The trial of the second generation DNMTi guadecitabine combined with colon vaccine (GVAX) secreting GM-CSF in the treatment of advanced CRC showed that the treatment was well tolerated and had no accidental toxicity, but it is closely related to the order of administration sequence $(171,172)$. These drugs, which have entered into clinical trials, show the potential of targeting macrophages in the treatment of CRC metastasis.

\section{DISCUSSION}

Clinical studies have shown that patients with $\mathrm{mCRC}$ have short survival time and poor prognosis, which indicate that inhibiting the metastasis of CRC is the critical point to treatment (179). With the effective application of immune checkpoint inhibitors in the treatment of melanoma, the prospects of immunotherapy for the treatment of other cancer types, including CRC, have been gradually proposed. The inhibition of immune checkpoints can enhance the tumor immune response and inhibit tumor development. PD-1 and TIM-3 are two significant immunosuppressive molecules, which have a crucial effect on immune escape and tumor development (180). PD-1 exists on the surface of $\mathrm{T}$ lymphocytes and is bound by its ligand PD-L1 expressed on Tregs or tumor cells, causing the reduction of tumor immunity (181-183). PD-L1 expression is a suitable prognostic biomarker to predict the survival of patients with CRC. In stage I-III CRC patients, the upregulated expression of TIM-3 and PD-1 may predict poor prognosis (180). Furthermore, there are significant differences in expression between metastatic and primary tumors. PD-1 expression in tumor-infiltrating lymphocytes is a strong prognostic indicator for CRC patients following pulmonary resection for CRC metastasis (182). In addition, lymphocyte activation gene 3 (LAG3) is also an immune checkpoint protein (184). Blocking LAG3 can enhance tumor-infiltrating $\mathrm{T}$ cell response in patients with mismatch-repair proficient liver metastasis of CRC (185), which might be a newfound immunotherapy target for CRC liver metastasis. CTLA-4 expressed by $\mathrm{T}$ cells can also inhibit the activity of CD8+ $\mathrm{T}$ cells and tumor immunity. All the abovementioned proteins are important immune checkpoints, which can suppress $\mathrm{T}$ effector cell proliferation and consequently inhibit tumor immunity (186). Clinically, PD-1 inhibitors are effective in mCRC with mismatch repair defects and high microsatellite instability (dMMR-MSI-H), which provides a rationale for the development and application of immunotherapy in mCRC (187). As one of the most abundant immune cell types in the TIME, TAMs have fundamental significance for their development as potential targets in tumor therapy. Studies have shown that the phagocytic ability of PD-1+ TAMs is decreased, thus PD- 1 inhibitors also play a critical role in the targeted therapy of TAMs (173).

In TIME, some molecules, as cellular receptors, can directly target TAMs by inhibiting the development of CRC through their inhibitors. Some molecules, such as cytokines or noncoding RNA, that activate tumor-related signal pathways can promote the immunosuppression of TIME, increase the recruitment and infiltration of immunosuppressive cells, promote EMT and tumor angiogenesis, and indirectly promote the CRC metastasis through the crosstalk between macrophages and tumor cells. Also some molecules, as cell products, can induce the polarization of M2 macrophages and predict a poor prognosis in patients.

Researchers have identified a number of factors that regulate macrophages, and we have classified and summarized their findings. According to Table 1, we can regulate or block the molecules that interfere with TAM infiltration or M2-like polarization, so that their depletion and reprogramming can then inhibit CRC metastasis. At present, there are few studies on direct targeting of macrophages. With the rise of nanometer technology and its application in tumor treatment (188), we hypothesize that we can use the existing nanomaterial-targeting technology to identify the unique surface markers that directly and specifically bind to TAMs and to remove or block their synergistic invasion of CRC cells; however, this technology needs to be further investigated by researchers. Inhibitors or gene knockout methods can be used in in vivo and in vitro experiments to regulate related molecules, directly or indirectly inhibit the tumor-promoting effect of TAMs, and then induce targeted macrophages to interfere with the process of CRC metastasis.

At present, there are still numerous challenges for the development of macrophages as molecular targeted therapy for tumors. Most biomarkers associated with macrophages play an indirect role, but their effects are not completely clear. Furtherly, TAM-associated molecular targets and their therapeutic effects on CRC still require verification using experimental models. Tumor treatment that relates to macrophages has entered clinical application and the associated immunotherapeutic and targeted therapy has shown the effective potential to inhibit tumor metastasis, but its clinical application is still very limited and requires further exploration before its therapeutic benefits are expanded as an intervention for tumor metastasis.

Although targeted drug therapy has achieved a certain degree of therapeutic efficacy, these agents are not effective for all patients. Besides, prolonged treatment with targeted drugs may also result in drug resistance. Studies have shown that TAMs are associated with drug resistance to bevacizumab, and TAMs secrete IL- 8 which induces drug resistance to lapatinib by activating EGFR signaling (189). In a phase III clinical trial, the addition of cetuximab to mCRC patients who were treated with chemotherapy combined with bevacizumab activated M2 macrophages and reduced the progression-free survival rate (190). Thus, targeted drugs require further experimental evaluation despite their potential benefits for the treatment of cancers.

Targeted drug therapy is also limited by the degree of toxicity during treatment. Adverse effects mainly include skin toxicity and gastrointestinal reactions. Thus, the control of side effects is also a key point to be considered in the development of targeted 
drugs. Furthermore, exploration of therapeutic doses should also consider the maximal doses tolerated based on the condition of the patients in order to achieve the best therapeutic outcome and simultaneously to improve their quality of life during treatment. Therefore, before targeted drugs are fully applied in clinical practice, well-organized clinical trials are needed to fully elucidate the advantages of the approach and to determine ways to avoid side effects as much as possible. If these strategies can be applied to the human body after improvement, they can be used as supplementary strategies for routine treatment, which could prolong survival time and improve life quality of patients with advanced CRC.

In conclusion, CRC metastasis is a complex process associated with the interaction between tumor cells and their metastatic niche. In this paper, we described the feedback loop between CRC cells and TAMs in TIME during metastasis. As the main immune cells in the TIME, macrophages play a pivotal impact in the development of mCRC. Macrophages may exert tumoricidal effects as the M1 subtype and participate in tumor immunity. Conversely, macrophages also inhibit inflammatory reaction as in the M2 subtype and facilitate the development of mCRC. In the TIME of CRC, TAMs interact with cytokines, cell metabolites, and signaling pathways to regulate the TME of CRC.

\section{REFERENCES}

1. Keum N, Giovannucci E. Global Burden of Colorectal Cancer: Emerging Trends, Risk Factors and Prevention Strategies. Nat Rev Gastroenterol Hepatol (2019) 16(12):713-32. doi: 10.1038/s41575-019-0189-8

2. Silva-Fisher JM, Dang HX, White NM, Strand MS, Krasnick BA, Rozycki EB, et al. Long Non-Coding RNA RAMS11 Promotes Metastatic Colorectal Cancer Progression. Nat Commun (2020) 11(1):2156. doi: 10.1038/s41467020-15547-8

3. Zhang T, Guo J, Gu J, Wang Z, Wang G, Li H, et al. Identifying the Key Genes and microRNAs in Colorectal Cancer Liver Metastasis by Bioinformatics Analysis and In Vitro Experiments. Oncol Rep (2019) 41 (1):279-91. doi: 10.3892/or.2018.6840

4. Mitry E, Guiu B, Cosconea S, Jooste V, Faivre J, Bouvier AM. Epidemiology, Management and Prognosis of Colorectal Cancer With Lung Metastases: A 30-Year Population-Based Study. Gut (2010) 59(10):1383-8. doi: 10.1136/ gut.2010.211557

5. Chen R-X, Chen X, Xia L-P, Zhang J-X, Pan Z-Z, Ma X-D, et al. NMethyladenosine Modification of Circnsun2 Facilitates Cytoplasmic Export and Stabilizes HMGA2 to Promote Colorectal Liver Metastasis. Nat Commun (2019) 10(1):4695. doi: 10.1038/s41467-019-12651-2

6. Van Cutsem E, Danielewicz I, Saunders MP, Pfeiffer P, Argilés G, Borg C, et al. Trifluridine/tipiracil Plus Bevacizumab in Patients With Untreated Metastatic Colorectal Cancer Ineligible for Intensive Therapy: The Randomized TASCO1 Study. Ann Oncol: Off J Eur Soc Med Oncol (2020) 31(9):1160-8. doi: 10.1016/j.annonc.2020.05.024

7. Kopetz S, Grothey A, Yaeger R, Van Cutsem E, Desai J, Yoshino T, et al. Encorafenib, Binimetinib, and Cetuximab in V600E-Mutated Colorectal Cancer. N Engl J Med (2019) 381(17):1632-43. doi: 10.1056/NEJMoa1908075

8. Chen J, Dai J, Kang Z, Yang T, Zhao Q, Zheng J, et al. A Combinatorial Strategy for Overcoming Primary and Acquired Resistance of MEK Inhibition in Colorectal Cancer. Exp Cell Res (2020) 393(1):112060. doi: 10.1016/j.yexcr.2020.112060

9. Taghizadeh H, Mader RM, Müllauer L, Erhart F, Kautzky-Willer A, Prager GW. Precision Medicine for the Management of Therapy Refractory Colorectal Cancer. J Pers Med (2020) 10(4):272. doi: 10.3390/jpm10040272

10. Qi L, Zhang Y, Song F, Han Y, Ding Y. A Newly Identified Small Molecular Compound Acts as a Protein Kinase Inhibitor to Suppress Metastasis of
We summarized the biomolecular markers associated with macrophage activity in the mCRC TIME and provided an outline of the rationale for the development of novel molecular targeted therapy for mCRC. Accordingly, targeting TAMs is a promising strategy for CRC metastasis immunotherapy.

\section{AUTHOR CONTRIBUTIONS}

YinZ contributed to design the article structure, writing-review and editing, and draw the figures and tables. YiyZ contributed to writing-original draft and draw the figures and tables. QL contributed to conceptualization and funding acquisition. YW contributed to supervision, funding acquisition, and project administration. All authors contributed to the article and approved the submitted version.

\section{FUNDING}

This work was supported by the National Natural Science Foundation of China (81774095, 82074232, 82030118).
Colorectal Cancer. Bioorg Chem (2021) 107:104625. doi: 10.1016/ j.bioorg.2021.104625

11. Gao S, Li N, Gao S, Xue Q, Ying J, Wang S, et al. Neoadjuvant PD-1 Inhibitor (Sintilimab) in NSCLC. J Thorac Oncol (2020) 15(5):816-26. doi: 10.1016/ j.jtho.2020.01.017

12. Chalabi M, Fanchi LF, Dijkstra KK, Van den Berg JG, Aalbers AG, Sikorska $\mathrm{K}$, et al. Neoadjuvant Immunotherapy Leads to Pathological Responses in MMR-Proficient and MMR-Deficient Early-Stage Colon Cancers. Nat Med (2020) 26(4):566-76. doi: 10.1038/s41591-020-0805-8

13. Pitt JM, Marabelle A, Eggermont A, Soria JC, Kroemer G, Zitvogel L. Targeting the Tumor Microenvironment: Removing Obstruction to Anticancer Immune Responses and Immunotherapy. Ann Oncol (2016) 27 (8):1482-92. doi: 10.1093/annonc/mdw168

14. Wu J-Y, Huang T-W, Hsieh Y-T, Wang Y-F, Yen C-C, Lee G-L, et al. Cancer-Derived Succinate Promotes Macrophage Polarization and Cancer Metastasis via Succinate Receptor. Mol Cell (2020) 77(2):213-27.e5. doi: 10.1016/j.molcel.2019.10.023

15. Wang X, Luo G, Zhang K, Cao J, Huang C, Jiang T, et al. Hypoxic TumorDerived Exosomal miR-301a Mediates M2 Macrophage Polarization via PTEN/Pi3k $\gamma$ to Promote Pancreatic Cancer Metastasis. Cancer Res (2018) 78 (16):4586-98. doi: 10.1158/0008-5472.CAN-17-3841

16. Xia Y, Rao L, Yao H, Wang Z, Ning P, Chen X. Engineering Macrophages for Cancer Immunotherapy and Drug Delivery. Adv Mater (Deerfield Beach Fla) (2020) 32(40):e2002054. doi: 10.1158/0008-5472.CAN-17-3841

17. Wang H, Wang X, Xu L, Zhang J, Cao H. Analysis of the Transcriptomic Features of Microsatellite Instability Subtype Colon Cancer. BMC Cancer (2019) 19(1):605. doi: 10.1186/s12885-019-5802-2

18. van den Ende T, van den Boorn HG, Hoonhout NM, van Etten-Jamaludin FS, Meijer SL, Derks S, et al. Priming the Tumor Immune Microenvironment With Chemo(Radio)Therapy: A Systematic Review Across Tumor Types. Biochim Biophys Acta Rev Cancer (2020) 1874 (1):188386. doi: 10.1016/j.bbcan.2020.188386

19. Huang J, Li J, Zheng S, Lu Z, Che Y, Mao S, et al. Tumor Microenvironment Characterization Identifies Two Lung Adenocarcinoma Subtypes With Specific Immune and Metabolic State. Cancer Sci (2020) 111(6):1876-86. doi: $10.1111 /$ cas. 14390

20. Szabo PA, Levitin HM, Miron M, Snyder ME, Senda T, Yuan J, et al. SingleCell Transcriptomics of Human T Cells Reveals Tissue and Activation 
Signatures in Health and Disease. Nat Commun (2019) 10(1):4706. doi: 10.1038/s41467-019-12464-3

21. Park SM, Do-Thi VA, Lee J-O, Lee H, Kim YS. Interleukin-9 Inhibits Lung Metastasis of Melanoma Through Stimulating Anti-Tumor M1 Macrophages. Mol Cells (2020) 43(5):479-90. doi: 10.14348/molcells. 2020.0047

22. Sanchez-Correa B, Valhondo I, Hassouneh F, Lopez-Sejas N, Pera A, Bergua JM, et al. DNAM-1 and the TIGIT/PVRIG/TACTILE Axis: Novel Immune Checkpoints for Natural Killer Cell-Based Cancer Immunotherapy. Cancers (2019) 11(6):877. doi: 10.3390/cancers11060877

23. Arlauckas SP, Garren SB, Garris CS, Kohler RH, Oh J, Pittet MJ, et al. Arg1 Expression Defines Immunosuppressive Subsets of Tumor-Associated Macrophages. Theranostics (2018) 8(21):5842-54. doi: 10.7150/thno.26888

24. Tavazoie MF, Pollack I, Tanqueco R, Ostendorf BN, Reis BS, Gonsalves FC, et al. LXR/ApoE Activation Restricts Innate Immune Suppression in Cancer. Cell (2018) 172(4):825-40.e18. doi: 10.1016/j.cell.2017.12.026

25. Liu C, Chikina M, Deshpande R, Menk AV, Wang T, Tabib T, et al. Treg Cells Promote the SREBP1-Dependent Metabolic Fitness of Tumor-Promoting Macrophages via Repression of CD8 T Cell-Derived Interferon- $\gamma$. Immunity (2019) 51(2):381-97.e6. doi: 10.1016/j.immuni.2019.06.017

26. Derynck R, Turley SJ, Akhurst RJ. Tgf $\beta$ Biology in Cancer Progression and Immunotherapy. Nat Rev Clin Oncol (2021) 18(1):9-34. doi: 10.1038/ s41571-020-0403-1

27. Liu J, Wu S, Zheng X, Zheng P, Fu Y, Wu C, et al. Immune Suppressed Tumor Microenvironment by Exosomes Derived From Gastric Cancer Cells via Modulating Immune Functions. Sci Rep (2020) 10(1):14749. doi: 10.1038/s41598-020-71573-y

28. Rosalia RA, Quakkelaar ED, Redeker A, Khan S, Camps M, Drijfhout JW, et al. Dendritic Cells Process Synthetic Long Peptides Better Than Whole Protein, Improving Antigen Presentation and T-Cell Activation. Eur J Immunol (2013) 43(10):2554-65. doi: 10.1002/eji.201343324

29. Xie Z, Zheng J, Wang Y, Li D, Maermaer T, Li Y, et al. Deficient IL-2 Produced by Activated CD56 T Cells Contributes to Impaired NK CellMediated ADCC Function in Chronic HIV-1 Infection. Front Immunol (2019) 10:1647. doi: 10.3389/fimmu.2019.01647

30. Su S, Zhao J, Xing Y, Zhang X, Liu J, Ouyang Q, et al. Immune Checkpoint Inhibition Overcomes ADCP-Induced Immunosuppression by Macrophages. Cell (2018) 175(2):442-57.e23. doi: 10.1016/j.cell.2018.09.007

31. Lee S-H, Bar-Haim E, Machlenkin A, Goldberger O, Volovitz I, Vadai E, et al. In Vivo Rejection of Tumor Cells Dependent on CD8 Cells That Kill Independently of Perforin and FasL. Cancer Gene Ther (2004) 11(3):237-48. doi: $10.1038 /$ sj.cgt.7700678

32. Kearney CJ, Vervoort SJ, Hogg SJ, Ramsbottom KM, Freeman AJ, Lalaoui N, et al. Tumor Immune Evasion Arises Through Loss of TNF Sensitivity. Sci Immunol (2018) 3(23):eaar3451. doi: 10.1126/sciimmunol.aar3451

33. Beury DW, Parker KH, Nyandjo M, Sinha P, Carter KA, Ostrand-Rosenberg S. Cross-Talk Among Myeloid-Derived Suppressor Cells, Macrophages, and Tumor Cells Impacts the Inflammatory Milieu of Solid Tumors. J Leukoc Biol (2014) 96(6):1109-18. doi: 10.1189/jlb.3A0414-210R

34. Huang B, Pan PY, Li Q, Sato AI, Levy DE, Bromberg J, et al. Gr-1+CD115+ Immature Myeloid Suppressor Cells Mediate the Development of TumorInduced T Regulatory Cells and T-Cell Anergy in Tumor-Bearing Host. Cancer Res (2006) 66(2):1123-31. doi: 10.1158/0008-5472.CAN-05-1299

35. Mandai M, Hamanishi J, Abiko K, Matsumura N, Baba T, Konishi I. Dual Faces of IFN $\gamma$ in Cancer Progression: A Role of PD-L1 Induction in the Determination of Pro- and Antitumor Immunity. Clin Cancer Res (2016) 22 (10):2329-34. doi: 10.1158/1078-0432.CCR-16-0224

36. Syed Khaja AS, Toor SM, El Salhat H, Ali BR, Elkord E. Intratumoral FoxP3 $(+)$ Helios(+) Regulatory T Cells Upregulating Immunosuppressive Molecules Are Expanded in Human Colorectal Cancer. Front Immunol (2017) 8:619. doi: 10.3389/fimmu.2017.00619

37. Zhang X, Li W, Sun J, Yang Z, Guan Q, Wang R, et al. How to Use Macrophages to Realise the Treatment of Tumour. J Drug Target (2020) 28 (10):1034-45. doi: 10.1080/1061186X.2020.1775236

38. Väyrynen JP, Haruki K, Lau MC, Väyrynen SA, Zhong R, Dias Costa A, et al. The Prognostic Role of Macrophage Polarization in the Colorectal Cancer Microenvironment. Cancer Immunol Res (2021) 9(1):8-19. doi: 10.1158/ 2326-6066.CIR-20-0527
39. Ma H, Li Y-N, Song L, Liu R, Li X, Shang Q, et al. Macrophages Inhibit Adipogenic Differentiation of Adipose Tissue Derived Mesenchymal Stem/ Stromal Cells by Producing Pro-Inflammatory Cytokines. Cell Biosci (2020) 10:88. doi: 10.1186/s13578-020-00450-y

40. Quero L, Hanser E, Manigold T, Tiaden AN, Kyburz D. TLR2 Stimulation Impairs Anti-Inflammatory Activity of M2-Like Macrophages, Generating a Chimeric M1/M2 Phenotype. Arthritis Res Ther (2017) 19(1):245. doi: 10.1186/s13075-017-1447-1

41. Ma M, Wang X, Liu N, Shan F, Feng Y. Low-Dose Naltrexone Inhibits Colorectal Cancer Progression and Promotes Apoptosis by Increasing M1Type Macrophages and Activating the Bax/Bcl-2/Caspase-3/PARP Pathway. Int Immunopharmacol (2020) 83:106388. doi: 10.1016/j.intimp.2020.106388

42. Sun Y, Diao F, Niu Y, Li X, Zhou H, Mei Q, et al. Apple Polysaccharide Prevents From Colitis-Associated Carcinogenesis Through Regulating Macrophage Polarization. Int J Biol Macromol (2020) 161:704-11. doi: $10.1016 /$ j.ijbiomac.2020.06.121

43. Ma PF, Gao CC, Yi J, Zhao JL, Liang SQ, Zhao Y, et al. Cytotherapy With M1-Polarized Macrophages Ameliorates Liver Fibrosis by Modulating Immune Microenvironment in Mice. J Hepatol (2017) 67(4):770-9. doi: 10.1016/j.jhep.2017.05.022

44. Shi Y, Luo P, Wang W, Horst K, Bläsius F, Relja B, et al. M1 But Not M0 Extracellular Vesicles Induce Polarization of RAW264.7 Macrophages Via the TLR4-NFKB Pathway In Vitro. Inflammation (2020) 43(5):1611-9. doi: 10.1007/s10753-020-01236-7

45. Zhang F, Parayath NN, Ene CI, Stephan SB, Koehne AL, Coon ME, et al. Genetic Programming of Macrophages to Perform Anti-Tumor Functions Using Targeted mRNA Nanocarriers. Nat Commun (2019) 10(1):3974. doi: 10.1038/s41467-019-11911-5

46. Lin Y, Zhao JL, Zheng QJ, Jiang X, Tian J, Liang SQ, et al. Notch Signaling Modulates Macrophage Polarization and Phagocytosis Through Direct Suppression of Signal Regulatory Protein $\alpha$ Expression. Front Immunol (2018) 9:1744. doi: 10.3389/fimmu.2018.01744

47. Rösner T, Kahle S, Montenegro F, Matlung HL, Jansen JHM, Evers M, et al. Immune Effector Functions of Human IgG2 Antibodies Against EGFR. Mol Cancer Ther (2019) 18(1):75-88. doi: 10.1158/1535-7163.MCT-18-0341

48. Ramesh A, Kumar S, Brouillard A, Nandi D, Kulkarni A. A Nitric Oxide (NO) Nanoreporter for Noninvasive Real-Time Imaging of Macrophage Immunotherapy. Adv Mater (2020) 32(24):e2000648. doi: 10.1002/ adma.202000648

49. Baer C, Squadrito ML, Laoui D, Thompson D, Hansen SK, Kiialainen A, et al. Suppression of microRNA Activity Amplifies IFN- $\gamma$-Induced Macrophage Activation and Promotes Anti-Tumour Immunity. Nat Cell Biol (2016) 18(7):790-802. doi: 10.1038/ncb3371

50. Lee C, Jeong H, Bae Y, Shin K, Kang S, Kim H, et al. Targeting of M2-Like Tumor-Associated Macrophages With a Melittin-Based Pro-Apoptotic Peptide. J Immunother Cancer (2019) 7(1):147. doi: 10.1186/s40425-019-0610-4

51. Oishi S, Takano R, Tamura S, Tani S, Iwaizumi M, Hamaya Y, et al. M2 Polarization of Murine Peritoneal Macrophages Induces Regulatory Cytokine Production and Suppresses T-Cell Proliferation. Immunology (2016) 149(3):320-8. doi: 10.1111/imm.12647

52. Su B, Han H, Gong Y, Li X, Ji C, Yao J, et al. Let-7d Inhibits Intratumoral Macrophage M2 Polarization and Subsequent Tumor Angiogenesis by Targeting IL-13 and IL-10. Cancer Immunol Immunother (2020) 70 (6):1619-34. doi: 10.1007/s00262-020-02791-6

53. Dou C, Ding N, Zhao C, Hou T, Kang F, Cao Z, et al. Estrogen DeficiencyMediated M2 Macrophage Osteoclastogenesis Contributes to M1/M2 Ratio Alteration in Ovariectomized Osteoporotic Mice. J Bone Miner Res (2018) 33 (5):899-908. doi: 10.1002/jbmr.3364

54. Sui H, Tan H, Fu J, Song Q, Jia R, Han L, et al. The Active Fraction of Garcinia Yunnanensis Suppresses the Progression of Colorectal Carcinoma by Interfering With Tumorassociated Macrophage-Associated M2 Macrophage Polarization In Vivo and In Vitro. FASEB J: Off Publ Fed Am Soc Exp Biol (2020) 34(6):7387-403. doi: 10.1096/fj.201903011R

55. Wang Y, Wei B, Gao J, Cai X, Xu L, Zhong H, et al. Combination of Fruquintinib and Anti-PD-1 for the Treatment of Colorectal Cancer. J Immunol (2020) 205(10):2905-15. doi: 10.4049/jimmunol.2000463

56. Kim Y, Wen X, Bae JM, Kim JH, Cho NY, Kang GH. The Distribution of Intratumoral Macrophages Correlates With Molecular Phenotypes and 
Impacts Prognosis in Colorectal Carcinoma. Histopathology (2018) 73 (4):663-71. doi: 10.1111/his.13674

57. Min AKT, Mimura K, Nakajima S, Okayama H, Saito K, Sakamoto W, et al. Therapeutic Potential of Anti-VEGF Receptor 2 Therapy Targeting for M2Tumor-Associated Macrophages in Colorectal Cancer. Cancer Immunol Immunother (2021) 70(2):289-98. doi: 10.1007/s00262-020-02676-8

58. Ye YC, Zhao JL, Lu YT, Gao CC, Yang Y, Liang SQ, et al. NOTCH Signaling via WNT Regulates the Proliferation of Alternative, CCR2-Independent Tumor-Associated Macrophages in Hepatocellular Carcinoma. Cancer Res (2019) 79(16):4160-72. doi: 10.1158/0008-5472.CAN-18-1691

59. Zhu D, Johnson TK, Wang Y, Thomas M, Huynh K, Yang Q, et al. Macrophage M2 Polarization Induced by Exosomes From AdiposeDerived Stem Cells Contributes to the Exosomal Proangiogenic Effect on Mouse Ischemic Hindlimb. Stem Cell Res Ther (2020) 11(1):162. doi: 10.1186/s13287-020-01669-9

60. Ding J, Yang C, Zhang Y, Wang J, Zhang S, Guo D, et al. M2 MacrophageDerived G-CSF Promotes Trophoblasts EMT, Invasion and Migration via Activating PI3K/Akt/Erk1/2 Pathway to Mediate Normal Pregnancy. J Cell Mol Med (2021) 25(4):2136-47. doi: 10.1111/jcmm.16191

61. Wang Z, Du Z, Sheng H, Xu X, Wang W, Yang J, et al. Polarization of Intestinal Tumour-Associated Macrophages Regulates the Development of Schistosomal Colorectal Cancer. J Cancer (2021) 12(4):1033-41. doi: 10.7150/jca.48985

62. Wu MF, Lin CA, Yuan TH, Yeh HY, Su SF, Guo CL, et al. The M1/M2 Spectrum and Plasticity of Malignant Pleural Effusion-Macrophage in Advanced Lung Cancer. Cancer Immunol Immunother (2021) 70(5):143550. doi: $10.1007 / \mathrm{s} 00262-020-02781-8$

63. Cassetta L, Pollard JW. Tumor-Associated Macrophages. Curr Biol (2020) 30 (6):R246-R8. doi: 10.1007/s00262-020-02781-8

64. Mougiakakos D, Bach C, Böttcher M, Beier F, Röhner L, Stoll A, et al. The IKZF1-IRF4/IRF5 Axis Controls Polarization of Myeloma-Associated Macrophages. Cancer Immunol Res (2021) 9(3):265-78. doi: 10.1158/23266066.CIR-20-0555

65. Nie W, Wu G, Zhang J, Huang LL, Ding J, Jiang A, et al. Responsive Exosome Nano-Bioconjugates for Synergistic Cancer Therapy. Angew Chem (Int Ed English) (2020) 59(5):2018-22. doi: 10.1002/anie.201912524

66. Anastas JN, Moon RT. WNT Signalling Pathways as Therapeutic Targets in Cancer. Nat Rev Cancer (2013) 13(1):11-26. doi: 10.1038/nrc3419

67. Parker TW, Neufeld KL. APC Controls Wnt-Induced Beta-Catenin Destruction Complex Recruitment in Human Colonocytes. Sci Rep (2020) 10(1):2957. doi: 10.1038/s41598-020-59899-z

68. Soldevilla B, Carretero-Puche C, Gomez-Lopez G, Al-Shahrour F, Riesco MC, Gil-Calderon B, et al. The Correlation Between Immune Subtypes and Consensus Molecular Subtypes in Colorectal Cancer Identifies Novel Tumour Microenvironment Profiles, With Prognostic and Therapeutic Implications. Eur J Cancer (Oxf Engl 1990) (2019) 123:118-29. doi: 10.1016/j.ejca.2019.09.008

69. Wang B, Tian T, Kalland KH, Ke X, Qu Y. Targeting Wnt/beta-Catenin Signaling for Cancer Immunotherapy. Trends Pharmacol Sci (2018) 39 (7):648-58. doi: 10.1016/j.tips.2018.03.008

70. Metzger R, Maruskova M, Krebs S, Janssen KP, Krug AB. Increased Incidence of Colon Tumors in AOM-Treated Apc (1638n/+) Mice Reveals Higher Frequency of Tumor Associated Neutrophils in Colon Than Small Intestine. Front Oncol (2019) 9:1001. doi: 10.3389/fonc.2019.01001

71. Buhrmann C, Shayan P, Banik K, Kunnumakkara AB, Kubatka P, Koklesova $\mathrm{L}$, et al. Targeting NF- $\mathrm{\kappa B}$ Signaling by Calebin A, a Compound of Turmeric, in Multicellular Tumor Microenvironment: Potential Role of Apoptosis Induction in CRC Cells. Biomedicines (2020) 8(8):236. doi: 10.3390/ biomedicines8080236

72. Yang Y, Weng W, Peng J, Hong L, Yang L, Toiyama Y, et al. Fusobacterium Nucleatum Increases Proliferation of Colorectal Cancer Cells and Tumor Development in Mice by Activating Toll-Like Receptor 4 Signaling to Nuclear Factor- $\mathrm{\kappa B}$, and Up-Regulating Expression of MicroRNA-21. Gastroenterology (2017) 152(4):851-66.e24. doi: 10.1053/j.gastro.2016. 11.018

73. Wu Y, Konaté MM, Lu J, Makhlouf H, Chuaqui R, Antony S, et al. IL-4 and IL-17a Cooperatively Promote Hydrogen Peroxide Production, Oxidative DNA Damage, and Upregulation of Dual Oxidase 2 in Human Colon and
Pancreatic Cancer Cells. J Immunol (2019) 203(9):2532-44. doi: 10.4049/ jimmunol.1800469

74. Huang M, Wu R, Chen L, Peng Q, Li S, Zhang Y, et al. S100A9 Regulates MDSCs-Mediated Immune Suppression via the RAGE and TLR4 Signaling Pathways in Colorectal Carcinoma. Front Immunol (2019) 10:2243. doi: 10.3389/fimmu.2019.02243

75. Sasidharan Nair V, Toor S M, Taha R Z, Ahmed AA, Kurer MA, Murshed K, et al. Transcriptomic Profiling of Tumor-Infiltrating CD4+TIM-3+ T Cells Reveals Their Suppressive, Exhausted, and Metastatic Characteristics in Colorectal Cancer Patients. Vaccines (2020) 8(1). doi: 10.3390/ vaccines 8010071

76. Yang C, Shi S, Su Y, Tong JS, Li L. P2X7R Promotes Angiogenesis and Tumour-Associated Macrophage Recruitment by Regulating the NF- $\mathrm{KB}$ Signalling Pathway in Colorectal Cancer Cells. J Cell Mol Med (2020) 24 (18):10830-41. doi: 10.1111/jcmm.15708

77. Zhang T, Liu L, Lai W, Zeng Y, Xu H, Lan Q, et al. Interaction With TumorAssociated Macrophages Promotes PRL-3-induced Invasion of Colorectal Cancer Cells via MAPK Pathway-Induced EMT and NF-KB Signaling-Induced Angiogenesis. Oncol Rep (2019) 41(5):2790-802. doi: 10.3892/or.2019.7049

78. Porta C, Ippolito A, Consonni FM, Carraro L, Celesti G, Correale C, et al. Protumor Steering of Cancer Inflammation by P50 NF-KB Enhances Colorectal Cancer Progression. Cancer Immunol Res (2018) 6(5):578-93. doi: 10.1158/2326-6066.CIR-17-0036

79. Giampazolias E, Zunino B, Dhayade S, Bock F, Cloix C, Cao K, et al. Mitochondrial Permeabilization Engages NF- $\mathrm{kB}$-Dependent Anti-Tumour Activity Under Caspase Deficiency. Nat Cell Biol (2017) 19(9):1116-29. doi: $10.1038 /$ ncb3596

80. Johnson DE, O'Keefe RA, Grandis JR. Targeting the IL-6/JAK/STAT3 Signalling Axis in Cancer. Nat Rev Clin Oncol (2018) 15(4):234-48. doi: 10.1038/nrclinonc.2018.8

81. Zhong Q, Fang Y, Lai Q, Wang S, He C, Li A, et al. CPEB3 Inhibits Epithelial-Mesenchymal Transition by Disrupting the Crosstalk Between Colorectal Cancer Cells and Tumor-Associated Macrophages via IL-6r/ STAT3 Signaling. J Exp Clin Cancer Res: CR (2020) 39(1):132. doi: 10.1186/s13046-020-01637-4

82. Wei C, Yang C, Wang S, Shi D, Zhang C, Lin X, et al. Crosstalk Between Cancer Cells and Tumor Associated Macrophages is Required for Mesenchymal Circulating Tumor Cell-Mediated Colorectal Cancer Metastasis. Mol Cancer (2019) 18(1):64. doi: 10.1186/s12943-019-0976-4

83. Walens A, DiMarco AV, Lupo R, Kroger BR, Damrauer JS, Alvarez JV. CCL5 Promotes Breast Cancer Recurrence Through Macrophage Recruitment in Residual Tumors. eLife (2019) 8:e43653. doi: 10.7554/eLife.43653

84. Wang X, Yang X, Tsai Y, Yang L, Chuang KH, Keng PC, et al. IL-6 Mediates Macrophage Infiltration After Irradiation via Up-Regulation of CCL2/CCL5 in Non-Small Cell Lung Cancer. Radiat Res (2017) 187(1):50-9. doi: $10.1667 / R R 14503.1$

85. Wang S, Liang K, Hu Q, Li P, Song J, Yang Y, et al. JAK2-Binding Long Noncoding RNA Promotes Breast Cancer Brain Metastasis. J Clin Invest (2017) 127(12):4498-515. doi: 10.1172/JCI91553

86. Chun E, Lavoie S, Michaud M, Gallini CA, Kim J, Soucy G, et al. CCL2 Promotes Colorectal Carcinogenesis by Enhancing Polymorphonuclear Myeloid-Derived Suppressor Cell Population and Function. Cell Rep (2015) 12(2):244-57. doi: 10.1016/j.celrep.2015.06.024

87. Janku F, Yap TA, Meric-Bernstam F. Targeting the PI3K Pathway in Cancer: Are We Making Headway? Nat Rev Clin Oncol (2018) 15(5):273-91. doi: $10.1038 /$ nrclinonc. 2018.28

88. Hoxhaj G, Manning BD. The PI3K-AKT Network at the Interface of Oncogenic Signalling and Cancer Metabolism. Nat Rev Cancer (2020) 20 (2):74-88. doi: 10.1038/s41568-019-0216-7

89. Ren F, Zhao Q, Liu B, Sun X, Tang Y, Huang H, et al. Transcriptome Analysis Reveals GPNMB as a Potential Therapeutic Target for Gastric Cancer. J Cell Physiol (2020) 235(3):2738-52. doi: 10.1002/jcp.29177

90. Zhao R, Song Y, Wang Y, Huang Y, Li Z, Cui Y, et al. PD-1/PD-L1 Blockade Rescue Exhausted CD8+ T Cells in Gastrointestinal Stromal Tumours via the PI3K/Akt/mTOR Signalling Pathway. Cell Prolif (2019) 52(3):e12571. doi: $10.1111 /$ cpr.12571

91. Tan X, Zhang Z, Yao H, Shen L. Tim-4 Promotes the Growth of Colorectal Cancer by Activating Angiogenesis and Recruiting Tumor-Associated 
Macrophages via the PI3K/AKT/mTOR Signaling Pathway. Cancer Lett (2018) 436:119-28. doi: 10.1016/j.canlet.2018.08.012

92. Wang D, Wang X, Si M, Yang J, Sun S, Wu H, et al. Exosome-Encapsulated miRNAs Contribute to CXCL12/CXCR4-Induced Liver Metastasis of Colorectal Cancer by Enhancing M2 Polarization of Macrophages. Cancer Lett (2020) 474:36-52. doi: 10.1016/j.canlet.2020.01.005

93. Klebanoff CA, Crompton JG, Leonardi AJ, Yamamoto TN, Chandran SS, Eil RL, et al. Inhibition of AKT Signaling Uncouples T Cell Differentiation From Expansion for Receptor-Engineered Adoptive Immunotherapy. JCI Insight (2017) 2(23):e95103. doi: 10.1172/jci.insight.95103

94. Meng X, Zhang S. MAPK Cascades in Plant Disease Resistance Signaling. Annu Rev Phytopathol (2013) 51:245-66. doi: 10.1146/annurev-phyto082712-102314

95. Sun H, Ou B, Zhao S, Liu X, Song L, Liu X, et al. USP11 Promotes Growth and Metastasis of Colorectal Cancer via PPP1CA-Mediated Activation of ERK/MAPK Signaling Pathway. EBioMedicine (2019) 48:236-47. doi: 10.1016/j.ebiom.2019.08.061

96. Yang MH, Zhao L, Wang L, Ou-Yang W, Hu SS, Li WL, et al. Nuclear IncRNA HOXD-AS1 Suppresses Colorectal Carcinoma Growth and Metastasis via Inhibiting HOXD3-Induced Integrin $\beta 3$ Transcriptional Activating and MAPK/AKT Signalling. Mol Cancer (2019) 18(1):31. doi: 10.1186/s12943-019-0955-9

97. Zhao H, Xu Z, Qin H, Gao Z, Gao L. miR-30b Regulates Migration and Invasion of Human Colorectal Cancer via SIX1. Biochem J (2014) 460 (1):117-25. doi: 10.1042/BJ20131535

98. Xu H, Zhang Y, Peña MM, Pirisi L, Creek KE. Six1 Promotes Colorectal Cancer Growth and Metastasis by Stimulating Angiogenesis and Recruiting Tumor-Associated Macrophages. Carcinogenesis (2017) 38(3):281-92. doi: 10.1093/carcin/bgw121

99. Zhang D, Qiu X, Li J, Zheng S, Li L, Zhao H. TGF- $\beta$ Secreted by TumorAssociated Macrophages Promotes Proliferation and Invasion of Colorectal Cancer via miR-34a-VEGF Axis. Cell Cycle (2018) 17(24):2766-78. doi: 10.1080/15384101.2018.1556064

100. Yang C, Wei C, Wang S, Shi D, Zhang C, Lin X, et al. Elevated CD163 $(+) / C D 68(+)$ Ratio at Tumor Invasive Front is Closely Associated With Aggressive Phenotype and Poor Prognosis in Colorectal Cancer. Int J Biol Sci (2019) 15(5):984-98. doi: 10.7150/ijbs.29836

101. Cai J, Xia L, Li J, Ni S, Song H, Wu X. Tumor-Associated Macrophages Derived TGF- $\beta$-Induced Epithelial to Mesenchymal Transition in Colorectal Cancer Cells Through Smad2,3-4/Snail Signaling Pathway. Cancer Res Treat (2019) 51(1):252-66. doi: 10.4143/crt.2017.613

102. Fakih M, Ouyang C, Wang C, Tu TY, Gozo MC, Cho M, et al. Immune Overdrive Signature in Colorectal Tumor Subset Predicts Poor Clinical Outcome. J Clin Invest (2019) 129(10):4464-76. doi: 10.1172/JCI127046

103. McGeachy MJ, Bak-Jensen KS, Chen Y, Tato CM, Blumenschein W, McClanahan T, et al. TGF-Beta and IL-6 Drive the Production of IL-17 and IL-10 by T Cells and Restrain T(H)-17 Cell-Mediated Pathology. Nat Immunol (2007) 8(12):1390-7. doi: 10.1038/ni1539

104. Chung L, Thiele Orberg E, Geis AL, Chan JL, Fu K, DeStefano Shields CE, et al. Bacteroides Fragilis Toxin Coordinates a Pro-Carcinogenic Inflammatory Cascade via Targeting of Colonic Epithelial Cells. Cell Host Microbe (2018) 23(2):203-14.e5. doi: 10.1016/j.chom.2018.01.007

105. Zhang Q, Atsuta I, Liu S, Chen C, Shi S, Shi S, et al. IL-17-Mediated M1/M2 Macrophage Alteration Contributes to Pathogenesis of BisphosphonateRelated Osteonecrosis of the Jaws. Clin Cancer Res (2013) 19(12):3176-88. doi: 10.1158/1078-0432.CCR-13-0042

106. Yue K, Ma JL, Jiang T, Yue J, Sun SK, Shen JL, et al. LncRNA RPPH1 Predicts Poor Prognosis and Regulates Cell Proliferation and Migration by Repressing P21 Expression in Gastric Cancer. Eur Rev Med Pharmacol Sci (2020) 24 (21):11072-80. doi: 10.26355/eurrev_202011_23593

107. Liang Z-x, Liu H-s, Wang F-w, Xiong L, Zhou C, Hu T, et al. LncRNA RPPH1 Promotes Colorectal Cancer Metastasis by Interacting With TUBB3 and by Promoting Exosomes-Mediated Macrophage M2 Polarization. Cell Death Dis (2019) 10(11):829. doi: 10.1038/s41419-019-2077-0

108. Zhang J, Li S, Zhang X, Li C, Zhang J, Zhou W. LncRNA HLA-F-AS1 Promotes Colorectal Cancer Metastasis by Inducing PFN1 in Colorectal Cancer-Derived Extracellular Vesicles and Mediating Macrophage
Polarization. Cancer Gene Ther (2021) 2021:10.1038/s41417-020-00276-3. doi: 10.1038/s41417-020-00276-3

109. Lan J, Sun L, Xu F, Liu L, Hu F, Song D, et al. M2 Macrophage-Derived Exosomes Promote Cell Migration and Invasion in Colon Cancer. Cancer Res (2019) 79(1):146-58. doi: 10.1158/0008-5472.CAN-18-0014

110. Zhao S, Mi Y, Guan B, Zheng B, Wei P, Gu Y, et al. Tumor-Derived Exosomal miR-934 Induces Macrophage M2 Polarization to Promote Liver Metastasis of Colorectal Cancer. J Hematol Oncol (2020) 13(1):156. doi: 10.1186/s13045-020-00991-2

111. Akimoto M, Maruyama R, Takamaru H, Ochiya T, Takenaga K. Soluble IL33 Receptor Sst2 Inhibits Colorectal Cancer Malignant Growth by Modifying the Tumour Microenvironment. Nat Commun (2016) 7:13589. doi: 10.1038/ ncomms 13589

112. Liu Q, Song J, Pan Y, Shi D, Yang C, Wang S, et al. Wnt5a/CaMKII/ERK/CCL2 Axis Is Required for Tumor-Associated Macrophages to Promote Colorectal Cancer Progression. Int J Biol Sci (2020) 16(6):1023-34. doi: 10.7150/ijbs.40535

113. Liu C, Yao Z, Wang J, Zhang W, Yang Y, Zhang Y, et al. MacrophageDerived CCL5 Facilitates Immune Escape of Colorectal Cancer Cells via the P65/STAT3-CSN5-PD-L1 Pathway. Cell Death Differ (2020) 27(6):1765-81. doi: 10.1038/s41418-019-0460-0

114. Wang D, Sun H, Wei J, Cen B, DuBois RN. CXCL1 Is Critical for Premetastatic Niche Formation and Metastasis in Colorectal Cancer. Cancer Res (2017) 77(13):3655-65. doi: 10.1158/0008-5472.CAN-16-3199

115. Deng Q, Geng Y, Zhao L, Li R, Zhang Z, Li K, et al. NLRP3 Inflammasomes in Macrophages Drive Colorectal Cancer Metastasis to the Liver. Cancer Lett (2019) 442:21-30. doi: 10.1016/j.canlet.2018.10.030

116. Ohashi K, Wang Z, Yang YM, Billet S, Tu W, Pimienta M, et al. NOD-Like Receptor C4 Inflammasome Regulates the Growth of Colon Cancer Liver Metastasis in NAFLD. Hepatology (2019) 70(5):1582-99. doi: 10.1002/hep.30693

117. Liu Q, Yang C, Wang S, Shi D, Wei C, Song J, et al. Wnt5a-Induced M2 Polarization of Tumor-Associated Macrophages via IL-10 Promotes Colorectal Cancer Progression. Cell Commun Signal (2020) 18(1):51. doi: 10.1186/s12964-020-00557-2

118. Zang $\mathrm{Y}$, Dong Q, Lu Y, Dong K, Wang R, Liang Z. Lumican Inhibits Immune Escape and Carcinogenic Pathways in Colorectal Adenocarcinoma. Aging (Albany NY) (2021) 13(3):4388-408. doi: 10.18632/aging.202401

119. Shang S, Ji X, Zhang L, Chen J, Li C, Shi R, et al. Macrophage ABHD5 Suppresses NFKB-Dependent Matrix Metalloproteinase Expression and Cancer Metastasis. Cancer Res (2019) 79(21):5513-26. doi: 10.1158/00085472.CAN-19-1059

120. Alonso A, Sasin J, Bottini N, Friedberg I, Friedberg I, Osterman A, et al. Protein Tyrosine Phosphatases in the Human Genome. Cell (2004) 117 (6):699-711. doi: 10.1016/j.cell.2004.05.018

121. Lan Q, Lai W, Zeng Y, Liu L, Li S, Jin S, et al. CCL26 Participates in the PRL3-Induced Promotion of Colorectal Cancer Invasion by Stimulating TumorAssociated Macrophage Infiltration. Mol Cancer Ther (2018) 17(1):276-89. doi: 10.1158/1535-7163.MCT-17-0507

122. Ruess DA, Heynen GJ, Ciecielski KJ, Ai J, Berninger A, Kabacaoglu D, et al. Mutant KRAS-Driven Cancers Depend on PTPN11/SHP2 Phosphatase. Nat Med (2018) 24(7):954-60. doi: 10.1038/s41591-018-0024-8

123. Li R, Zhou R, Wang H, Li W, Pan M, Yao X, et al. Gut Microbiota-Stimulated Cathepsin K Secretion Mediates TLR4-Dependent M2 Macrophage Polarization and Promotes Tumor Metastasis in Colorectal Cancer. Cell Death Differ (2019) 26(11):2447-63. doi: 10.1038/s41418-019-0312-y

124. Zha H, Sun H, Li X, Duan L, Li A, Gu Y, et al. S100A8 Facilitates the Migration of Colorectal Cancer Cells Through Regulating Macrophages in the Inflammatory Microenvironment. Oncol Rep (2016) 36(1):279-90. doi: $10.3892 /$ or.2016.4790

125. Ayiomamitis GD, Notas G, Vasilakaki T, Tsavari A, Vederaki S, Theodosopoulos $\mathrm{T}$, et al. Understanding the Interplay Between COX-2 and hTERT in Colorectal Cancer Using a Multi-Omics Analysis. Cancers (Basel) (2019) 11(10):1536. doi: 10.3390/cancers11101536

126. Qian F, Xiao J, Hu B, Sun N, Yin W, Zhu J. High Expression of P2X7R is an Independent Postoperative Indicator of Poor Prognosis in Colorectal Cancer. Hum Pathol (2017) 64:61-8. doi: 10.1016/j.humpath.2017.03.019

127. Pan J-H, Zhou H, Cooper L, Huang J-L, Zhu S-B, Zhao X-X, et al. LAYN Is a Prognostic Biomarker and Correlated With Immune Infiltrates in Gastric 
and Colon Cancers. Front Immunol (2019) 10:6. doi: 10.3389/ fimmu.2019.00006

128. Lu W, Yu W, He J, Liu W, Yang J, Lin X, et al. Reprogramming Immunosuppressive Myeloid Cells Facilitates Immunotherapy for Colorectal Cancer. EMBO Mol Med (2021) 13(1):e12798. doi: 10.15252/ emmm.202012798

129. Bellamkonda K, Chandrashekar NK, Osman J, Selvanesan BC, Savari S, Sjölander A. The Eicosanoids Leukotriene D4 and Prostaglandin E2 Promote the Tumorigenicity of Colon Cancer-Initiating Cells in a Xenograft Mouse Model. BMC Cancer (2016) 16:425. doi: 10.1186/ s12885-016-2466-z

130. Campesato LF, Budhu S, Tchaicha J, Weng CH, Gigoux M, Cohen IJ, et al. Blockade of the AHR Restricts a Treg-Macrophage Suppressive Axis Induced by L-Kynurenine. Nat Commun (2020) 11(1):4011. doi: 10.1038/s41467-02017750-Z

131. Yang C, Dou R, Wei C, Liu K, Shi D, Zhang C, et al. Tumor-Derived Exosomal microRNA-106b-5p Activates EMT-Cancer Cell and M2-Subtype TAM Interaction to Facilitate CRC Metastasis. Mol Ther (2021). doi: 10.1016/j.ymthe.2021.02.006

132. Liu M, Sun T, Li N, Peng J, Fu D, Li W, et al. BRG1 Attenuates Colonic Inflammation and Tumorigenesis Through Autophagy-Dependent Oxidative Stress Sequestration. Nat Commun (2019) 10(1):4614. doi: 10.1038/s41467-019-12573-z

133. Metzger R, Maruskova M, Krebs S, Janssen K-P, Krug AB. Increased Incidence of Colon Tumors in AOM-Treated Mice Reveals Higher Frequency of Tumor Associated Neutrophils in Colon Than Small Intestine. Front Oncol (2019) 9:1001. doi: 10.3389/fonc.2019.01001

134. Liu C, Lu H, Wang H, Loo A, Zhang X, Yang G, et al. Combinations With Allosteric SHP2 Inhibitor TNO155 to Block Receptor Tyrosine Kinase Signaling. Clin Cancer Res (2021) 27(1):342-54. doi: 10.1158/10780432.CCR-20-2718

135. Nam SH, Kim D, Lee D, Lee H-M, Song D-G, Jung JW, et al. Lysyl-tRNA Synthetase-Expressing Colon Spheroids Induce M2 Macrophage Polarization to Promote Metastasis. J Clin Invest (2018) 128(11):5034-55. doi: 10.1172/JCI99806

136. Piao C, Zhang W-M, Li T-T, Zhang C-C, Qiu S, Liu Y, et al. Complement 5a Stimulates Macrophage Polarization and Contributes to Tumor Metastases of Colon Cancer. Exp Cell Res (2018) 366(2):127-38. doi: 10.1016/ j.yexcr.2018.03.009

137. Zhang L, Li Z, Ding G, La X, Yang P, Li Z. GRP78 Plays an Integral Role in Tumor Cell Inflammation-Related Migration Induced by M2 Macrophages. Cell Signal (2017) 37:136-48. doi: 10.1016/j.cellsig.2017.06.008

138. Herrero A, Benedicto A, Romayor I, Olaso E, Arteta B. Inhibition of COX-2 Impairs Colon Cancer Liver Metastasis Through Reduced Stromal Cell Reaction. Biomol Ther (Seoul) (2021) 29(3):342-51. doi: 10.4062/ biomolther.2020.160

139. Sharma RA, Gescher A, Plastaras JP, Leuratti C, Singh R, Gallacher-Horley B, et al. Cyclooxygenase-2, Malondialdehyde and Pyrimidopurinone Adducts of Deoxyguanosine in Human Colon Cells. Carcinogenesis (2001) 22 (9):1557-60. doi: 10.1093/carcin/22.9.1557

140. Huang GL, Tao A, Miyazaki T, Khan T, Hong T, Nakagawa Y, et al. PEGPoly(1-Methyl-L-Tryptophan)-Based Polymeric Micelles as Enzymatically Activated Inhibitors of Indoleamine 2,3-Dioxygenase. Nanomater (Basel) (2019) 9(5):719. doi: 10.3390/nano9050719

141. Wu D, Zhu Y. Role of Kynurenine in Promoting the Generation of Exhausted CD8+ T Cells in Colorectal Cancer. Am J Transl Res (2021) 13 (3):1535-47.

142. Huang Q, Xia J, Wang L, Wang X, Ma X, Deng Q, et al. miR-153 Suppresses IDO1 Expression and Enhances CAR T Cell Immunotherapy. J Hematol Oncol (2018) 11(1):58. doi: 10.1186/s13045-018-0600-x

143. Damato A, Iachetta F, Antonuzzo L, Nasti G, Bergamo F, Bordonaro R, et al. Phase II Study on First-Line Treatment of NIVolumab in Combination With Folfoxiri/Bevacizumab in Patients With Advanced COloRectal Cancer RAS or BRAF Mutated - NIVACOR Trial (GOIRC-03-2018). BMC Cancer (2020) 20(1):822. doi: 10.1186/s12885-020-07268-4

144. Osumi H, Shinozaki E, Ooki A, Wakatsuki T, Kamiimabeppu D, Sato T, et al. Early Hypertension and Neutropenia are Predictors of Treatment Efficacy in Metastatic Colorectal Cancer Patients Administered FOLFIRI and Vascular
Endothelial Growth Factor Inhibitors as Second-Line Chemotherapy. Cancer Med (2021) 10(2):615-25. doi: 10.1002/cam4.3638

145. Rahma OE, Hodi FS. The Intersection Between Tumor Angiogenesis and Immune Suppression. Clin Cancer Res (2019) 25(18):5449-57. doi: 10.1158/ 1078-0432.CCR-18-1543

146. Tsao LC, Crosby EJ, Trotter TN, Agarwal P, Hwang BJ, Acharya C, et al. CD47 Blockade Augmentation of Trastuzumab Antitumor Efficacy Dependent on Antibody-Dependent Cellular Phagocytosis. JCI Insight (2019) 4(24):e131882. doi: 10.1172/jci.insight.131882

147. Goldberg SB, Redman MW, Lilenbaum R, Politi K, Stinchcombe TE, Horn L, et al. Randomized Trial of Afatinib Plus Cetuximab Versus Afatinib Alone for First-Line Treatment of EGFR-Mutant Non-Small-Cell Lung Cancer: Final Results From SWOG S1403. J Clin Oncol: Off J Am Soc Clin Oncol (2020) 38(34):4076-85. doi: 10.1200/JCO.20.01149

148. Taniguchi H, Yamanaka T, Sakai D, Muro K, Yamazaki K, Nakata S, et al. Efficacy of Panitumumab and Cetuximab in Patients With Colorectal Cancer Previously Treated With Bevacizumab; a Combined Analysis of Individual Patient Data From ASPECCT and WJOG6510G. Cancers (Basel) (2020) 12 (7):1715. doi: $10.3390 /$ cancers 12071715

149. Zhao Y, Liu X, Huo M, Wang Y, Li Y, Xu N, et al. Cetuximab Enhances the Anti-Tumor Function of Macrophages in an IL-6 Dependent Manner. Life Sci (2021) 267:118953. doi: 10.1016/j.lfs.2020.118953

150. Sartore-Bianchi A, Trusolino L, Martino C, Bencardino K, Lonardi S, Bergamo F, et al. Dual-Targeted Therapy With Trastuzumab and Lapatinib in Treatment-Refractory, KRAS Codon 12/13 Wild-Type, HER2-Positive Metastatic Colorectal Cancer (HERACLES): A Proof-ofConcept, Multicentre, Open-Label, Phase 2 Trial. Lancet Oncol (2016) 17 (6):738-46. doi: 10.1016/S1470-2045(16)00150-9

151. Hannesdóttir L, Tymoszuk P, Parajuli N, Wasmer MH, Philipp S, Daschil N, et al. Lapatinib and Doxorubicin Enhance the Stat1-Dependent Antitumor Immune Response. Eur J Immunol (2013) 43(10):2718-29. doi: 10.1002/eji.201242505

152. Van Cutsem E, Martinelli E, Cascinu S, Sobrero A, Banzi M, Seitz JF, et al. Regorafenib for Patients With Metastatic Colorectal Cancer Who Progressed After Standard Therapy: Results of the Large, Single-Arm, Open-Label Phase IIIb CONSIGN Study. Oncol (2019) 24(2):185-92. doi: 10.1634/ theoncologist.2018-0072

153. Li J, Qin S, Xu R, Yau TC, Ma B, Pan H, et al. Regorafenib Plus Best Supportive Care Versus Placebo Plus Best Supportive Care in Asian Patients With Previously Treated Metastatic Colorectal Cancer (CONCUR): A Randomised, Double-Blind, Placebo-Controlled, Phase 3 Trial. Lancet Oncol (2015) 16(6):619-29. doi: 10.1016/S1470-2045(15)70156-7

154. Ou DL, Chen CW, Hsu CL, Chung CH, Feng ZR, Lee BS, et al. Regorafenib Enhances Antitumor Immunity via Inhibition of P38 Kinase/Creb1/Klf4 Axis in Tumor-Associated Macrophages. J Immunother Cancer (2021) 9(3): e001657. doi: 10.1136/jitc-2020-001657

155. Kim ST, Hong JY, Lee J, Park JO, Lim HY, Kang WK, et al. Pemetrexed/ Erlotinib as a Salvage Treatment in Patients With High EGFR-Expressing Metastatic Colorectal Cancer Following Failure of Standard Chemotherapy: A Phase II Single-Arm Prospective Study. Target Oncol (2020) 15(1):67-73. doi: 10.1007/s11523-019-00691-z

156. Grépin R, Guyot M, Dumond A, Durivault J, Ambrosetti D, Roussel JF, et al. The Combination of Bevacizumab/Avastin and Erlotinib/Tarceva Is Relevant for the Treatment of Metastatic Renal Cell Carcinoma: The Role of a Synonymous Mutation of the EGFR Receptor. Theranostics (2020) 10 (3):1107-21. doi: 10.7150/thno.38346

157. Shih CT, Shiau CW, Chen YL, Chen LJ, Chao TI, Wang CY, et al. TD-92, a Novel Erlotinib Derivative, Depletes Tumor-Associated Macrophages in Non-Small Cell Lung Cancer via Down-Regulation of CSF-1R and Enhances the Anti-Tumor Effects of Anti-PD-1. Cancer Lett (2021) 498:142-51. doi: 10.1016/j.canlet.2020.10.043

158. Jonker DJ, Nott L, Yoshino T, Gill S, Shapiro J, Ohtsu A, et al. Napabucasin Versus Placebo in Refractory Advanced Colorectal Cancer: A Randomised Phase 3 Trial. Lancet Gastroenterol Hepatol (2018) 3(4):263-70. doi: 10.1016/ S2468-1253(18)30009-8

159. Yin $\mathrm{Y}$, Yao $\mathrm{S}, \mathrm{Hu} \mathrm{Y}$, Feng $\mathrm{Y}, \mathrm{Li} \mathrm{M}$, Bian $\mathrm{Z}$, et al. The ImmuneMicroenvironment Confers Chemoresistance of Colorectal Cancer Through Macrophage-Derived Il6. Clin Cancer Res (2017) 23(23):7375-87. doi: 10.1158/1078-0432.CCR-17-1283 
160. Dienstmann R, Patnaik A, Garcia-Carbonero R, Cervantes A, Benavent M, Roselló S, et al. Safety and Activity of the First-In-Class Sym004 Anti-EGFR Antibody Mixture in Patients With Refractory Colorectal Cancer. Cancer Discovery (2015) 5(6):598-609. doi: 10.1158/2159-8290.CD-14-1432

161. Macarulla T, Cervantes A, Tabernero J, Roselló S, Van Cutsem E, Tejpar S, et al. Phase I Study of FOLFIRI Plus Pimasertib as Second-Line Treatment for KRAS-Mutated Metastatic Colorectal Cancer. Br J Cancer (2015) 112 (12):1874-81. doi: 10.1038/bjc.2015.144

162. Fane ME, Ecker BL, Kaur A, Marino GE, Alicea GM, Douglass SM, et al. Sfrp2 Supersedes VEGF as an Age-Related Driver of Angiogenesis in Melanoma, Affecting Response to Anti-VEGF Therapy in Older Patients. Clin Cancer Res (2020) 26(21):5709-19. doi: 10.1158/1078-0432.CCR-20-0446

163. Tabernero J, Hozak RR, Yoshino T, Cohn AL, Obermannova R, Bodoky G, et al. Analysis of Angiogenesis Biomarkers for Ramucirumab Efficacy in Patients With Metastatic Colorectal Cancer From RAISE, a Global, Randomized, Double-Blind, Phase III Study. Ann Oncol (2018) 29(3):6029. doi: 10.1093/annonc/mdx767

164. Liu X, Lukowski JK, Flinders C, Kim S, Georgiadis RA, Mumenthaler SM, et al. MALDI-MSI of Immunotherapy: Mapping the EGFR-Targeting Antibody Cetuximab in 3D Colon-Cancer Cell Cultures. Anal Chem (2018) 90(24):14156-64. doi: 10.1021/acs.analchem.8b02151

165. Lai YS, Wahyuningtyas R, Aui SP, Chang KT. Autocrine VEGF Signalling on M2 Macrophages Regulates PD-L1 Expression for Immunomodulation of T Cells. J Cell Mol Med (2019) 23(2):1257-67. doi: 10.1111/jcmm.14027

166. Hardbower DM, Coburn LA, Asim M, Singh K, Sierra JC, Barry DP, et al. EGFR-Mediated Macrophage Activation Promotes Colitis-Associated Tumorigenesis. Oncogene (2017) 36(27):3807-19. doi: 10.1038/onc.2017.23

167. Tamura R, Tanaka T, Morimoto Y, Kuranari Y, Yamamoto Y, Takei J, et al. Alterations of the Tumor Microenvironment in Glioblastoma Following Radiation and Temozolomide With or Without Bevacizumab. Ann Transl Med (2020) 8(6):297. doi: 10.21037/atm.2020.03.11

168. Ries CH, Cannarile MA, Hoves S, Benz J, Wartha K, Runza V, et al. Targeting Tumor-Associated Macrophages With Anti-CSF-1R Antibody Reveals a Strategy for Cancer Therapy. Cancer Cell (2014) 25(6):846-59. doi: 10.1016/j.ccr.2014.05.016

169. Available at: https://clinicaltrials.gov/ct2/show/study/NCT02777710?term= macrophages\&cond $=$ Colorectal + Cancer + Metastatic $\& d r a w=2 \&$ rank $=7$.

170. Park SH, Breitbach CJ, Lee J, Park JO, Lim HY, Kang WK, et al. Phase 1b Trial of Biweekly Intravenous Pexa-Vec (JX-594), an Oncolytic and Immunotherapeutic Vaccinia Virus in Colorectal Cancer. Mol Ther (2015) 23(9):1532-40. doi: 10.1038/mt.2015.109

171. Available at: https://clinicaltrials.gov/ct2/show/NCT01966289?term $=$ macrophages\&cond $=$ Colorectal + Cancer + Metastatic $\&$ draw $=2 \&$ rank $=3$.

172. Bever KM, Thomas DL2nd, Zhang J, Diaz Rivera EA, Rosner GL, Zhu Q, et al. A Feasibility Study of Combined Epigenetic and Vaccine Therapy in Advanced Colorectal Cancer With Pharmacodynamic Endpoint. Clin Epigenet (2021) 13(1):25. doi: 10.1186/s13148-021-01014-8

173. Gordon SR, Maute RL, Dulken BW, Hutter G, George BM, McCracken MN, et al. PD-1 Expression by Tumour-Associated Macrophages Inhibits Phagocytosis and Tumour Immunity. Nature (2017) 545(7655):495-9. doi: 10.1038/nature22396

174. Lin EY, Li JF, Gnatovskiy L, Deng Y, Zhu L, Grzesik DA, et al. Macrophages Regulate the Angiogenic Switch in a Mouse Model of Breast Cancer. Cancer Res (2006) 66(23):11238-46. doi: 10.1158/0008-5472.CAN-06-1278

175. Shi Y, Liu CH, Roberts AI, Das J, Xu G, Ren G, et al. Granulocyte-Macrophage Colony-Stimulating Factor (GM-CSF) and T-Cell Responses: What We do and Don't Know. Cell Res (2006) 16(2):126-33. doi: 10.1038/sj.cr.7310017

176. Available at: https://clinicaltrials.gov/ct2/show/NCT00656123?term= macrophages \& cond $=$ Colorectal + Cancer + Metastatic\&draw $=2 \&$ rank $=1$.

177. Zheng L, Edil BH, Soares KC, El-Shami K, Uram JN, Judkins C, et al. A Safety and Feasibility Study of an Allogeneic Colon Cancer Cell Vaccine Administered With a Granulocyte-Macrophage Colony Stimulating Factor-Producing
Bystander Cell Line in Patients With Metastatic Colorectal Cancer. Ann Surg Oncol (2014) 21(12):3931-7. doi: 10.1245/s10434-014-3844-x

178. Available at: https://clinicaltrials.gov/ct2/show/study/NCT01394939?term= macrophages\&cond $=$ Colorectal + Cancer + Metastatic $\& d r a w=2 \&$ rank $=10$.

179. Elez E, Chianese C, Sanz-García E, Martinelli E, Noguerido A, Mancuso FM, et al. Impact of Circulating Tumor DNA Mutant Allele Fraction on Prognosis in RAS-Mutant Metastatic Colorectal Cancer. Mol Oncol (2019) 13(9):1827-35. doi: 10.1002/1878-0261.12547

180. Kuai W, Xu X, Yan J, Zhao W, Li Y, Wang B, et al. Prognostic Impact of PD1 and Tim-3 Expression in Tumor Tissue in Stage I-III Colorectal Cancer. BioMed Res Int (2020) 2020:5294043. doi: 10.1155/2020/5294043

181. Yi M, Jiao D, Xu H, Liu Q, Zhao W, Han X, et al. Biomarkers for Predicting Efficacy of PD-1/PD-L1 Inhibitors. Mol Cancer (2018) 17(1):129. doi: 10.1186/s12943-018-0864-3

182. Kollmann D, Schweiger T, Schwarz S, Ignatova D, Chang YT, Lewik G, et al. PD1-Positive Tumor-Infiltrating Lymphocytes Are Associated With Poor Clinical Outcome After Pulmonary Metastasectomy for Colorectal Cancer. Oncoimmunology (2017) 6(9):e1331194. doi: 10.1080/2162402X.2017.1331194

183. Langhans B, Nischalke HD, Krämer B, Dold L, Lutz P, Mohr R, et al. Role of Regulatory T Cells and Checkpoint Inhibition in Hepatocellular Carcinoma. Cancer Immunol Immunother (2019) 68(12):2055-66. doi: 10.1007/s00262019-02427-4

184. Andrews LP, Marciscano AE, Drake CG, Vignali DAA. LAG3 (CD223) as a Cancer Immunotherapy Target. Immunol Rev (2017) 276(1):80-96. doi: 10.1111/imr.12519

185. Zhou G, Noordam L, Sprengers D, Doukas M, Boor PPC, van Beek AA, et al. Blockade of LAG3 Enhances Responses of Tumor-Infiltrating T Cells in Mismatch Repair-Proficient Liver Metastases of Colorectal Cancer. Oncoimmunology (2018) 7(7):e1448332. doi: 10.1080/2162402X.2018.1448332

186. Fu X, Luo H, Zheng Y, Wang S, Zhong Z, Wang Y, et al. CTLA-4 Immunotherapy Exposes Differences in Immune Response Along With Different Tumor Progression in Colorectal Cancer. Aging (Albany NY) (2020) 12(15):15656-69. doi: 10.18632/aging.103765

187. Overman MJ, McDermott R, Leach JL, Lonardi S, Lenz HJ, Morse MA, et al. Nivolumab in Patients With Metastatic DNA Mismatch Repair-Deficient or Microsatellite Instability-High Colorectal Cancer (CheckMate 142): An Open-Label, Multicentre, Phase 2 Study. Lancet Oncol (2017) 18(9):118291. doi: 10.1016/S1470-2045(17)30422-9

188. Zhang Y, Li C, Jia R, Gao R, Zhao Y, Ji Q, et al. PEG-Poly(Amino Acid)s/ EpCAM Aptamer Multifunctional Nanoparticles Arrest the Growth and Metastasis of Colorectal Cancer. Biomater Sci (2021) 9(10):3705-17. doi: 10.1039/d1bm00160d

189. Ahmed S, Mohamed HT, El-Husseiny N, El Mahdy MM, Safwat G, Diab AA, et al. IL-8 Secreted by Tumor Associated Macrophages Contribute to Lapatinib Resistance in HER2-Positive Locally Advanced Breast Cancer via Activation of Src/STAT3/ERK1/2-Mediated EGFR Signaling. Biochim Biophys Acta Mol Cell Res (2021) 1868(6):118995. doi: 10.1016/j.bbamcr.2021.118995

190. Pander J, Heusinkveld M, van der Straaten T, Jordanova ES, Baak-Pablo R, Gelderblom H, et al. Activation of Tumor-Promoting Type 2 Macrophages by EGFR-Targeting Antibody Cetuximab. Clin Cancer Res (2011) 17 (17):5668-73. doi: 10.1158/1078-0432.CCR-11-0239

Conflict of Interest: The authors declare that the research was conducted in the absence of any commercial or financial relationships that could be construed as a potential conflict of interest.

Copyright ( $\odot 2021$ Zhang, Zhao, Li and Wang. This is an open-access article distributed under the terms of the Creative Commons Attribution License (CC BY). The use, distribution or reproduction in other forums is permitted, provided the original author(s) and the copyright owner(s) are credited and that the original publication in this journal is cited, in accordance with accepted academic practice. No use, distribution or reproduction is permitted which does not comply with these terms. 